Año: 2020 Fecha de aceptado: 14/10/2019 Correo: revista.sciencia@up.ac.pa

Numero: 1 Número de páginas: 15-52 URL:https://revistas.up.ac.pa/index.php/scientia

\title{
SCOLYTINAE Y PLATYPODINAE (COLEOPTERA: CURCULIONIDAE) DE LA ISLA BARRO COLORADO, PANAMÁ.
}

\section{OSWALDO RODRÍGUEZ-FLORES ${ }^{1}$ y HECTOR BARRIOS ${ }^{2}$}

\author{
${ }^{1}$ Universidad Nacional Agraria, Departamento de Protección Agrícola y Forestal \\ Managua, Nicaragua \\ oroflores@yahoo.com \\ ${ }^{2}$ Universidad de Panamá, Programa Centroamericano de Maestría en Entomología, \\ Panamá, Panamá \\ hector.barriosv@up.ac.pa (autor para correspondencias)
}

\section{RESUMEN}

Isla Barro Colorado (BCI) es una reserva natural ubicada en el lago Gatún del Canal de Panamá, ha sido un área tropical importante para estudios en biología y ecología tropical. Sin embargo, ciertos grupos biológicos no cuentan con inventarios faunísticos, uno de ellos son los escarabajos ambrosiales (Coleoptera: Curculionidae). Por tal razón realizó un inventario enriquecido de los Scolytinae y Platypodinae reportados para BCI. Para desarrollar el inventario, se realizó: 1) revisión de literatura científica con registros de especies en la isla, 2) revisión de especímenes en colecciones entomológicas de la Universidad de Panamá y 3) identificación taxonómica de especímenes en muestras capturadas por el Instituto Smithsoniano de Investigaciones Tropicales. Un total de 48 especies de Scolytinae y cinco de Platypodinae se habían registrado para la isla en publicaciones científicas. La identificación taxonómica de muestras y especímenes en colecciones aportaron 64 especies de Scolytinae y tres de Platypodinae como nuevos registros para la isla. La cantidad de especies de Scolytinae conocidas para la isla alcanza las 113, mientras que los Platypodinae alcanzan 8; sin embargo, con la confirmación taxonómica de algunas especies, las cifras pueden aproximarse a 138 especies para Scolytinae y 20 para Platypodinae.

\section{PALABRAS ClAVES}


Escarabajos ambrosiales, Inventario taxonómico, Nuevos registros de insectos, Xilomicetófagia, Insectos cultivadores de hongos.

\section{INTRODUCCIÓN}

Los Scolytinae y Platypodinae son dos subfamilias de insectos coleópteros de la familia Curculionidae (Alonzo-Zarazaga y Lyal 2009). Ambos grupos insectiles son importantes componentes de las comunidades saproxílicas que descomponen e inician el reciclaje de los tejidos leñosos muertos en los bosques húmedos (Ulyshen et al., 2013). Los Platypodinae se encuentran entre los grupos dominantes de insectos en los bosques húmedos tropicales, la mayoría de las especies se encuentran en estos bosques (Jordal, 2015), mientras que los Scolytinae se encuentran en proporciones bastante iguales en todos los continentes boscosos, con la mayor diversidad en la zona tropical (Pistone et al., 2017).

Aunque son insectos de importancia ecológica, algunos de sus miembros pueden ser de importancia económica tanto en las regiones templadas como en las regiones tropicales (Beaver et al., 2016). De las dos subfamilias, los Scolytinae son los que tienen más especies de importancia económica, algunas especies se han vuelto invasivos y han causado grandes daños ecológicos y costos económicos (Hulcr y Dunn, 2011). Con respecto a los Platypodinae, son pocas las especies asociadas a daños en arboles (Ploetz et al., 2013). En américa se han reportado especies plagas de árboles forestales, como son: Euplatypus segnis como plaga del nogal (Soto et al., 2013; Atkinson, 2017), E. parallelus ataca una gran variedad de árboles (Cibrián et al., 1995, Beaver, 2013) y Megaplatypus mutatus en álamos y eucaliptos (Casaubon et al., 2006; Alfaro et al., 2007; Zanuncio et al., 2010).

La Isla Barro Colorado es una reserva natural ubicada en el lago Gatún del Canal de Panamá (Leigh y Wright, 1990). La isla, por medio de sus capacidades de investigación, es una de las áreas tropicales mejor conocida en materia de biología y ecología tropical (Leigh y Wright, 1990; Heckadon-Moreno et al., 1999). Basado en lo dicho anteriormente, la isla presenta características importantes, tres de ellas son su estabilidad ecológica, su ubicación geográfica (en un área de importancia internacional) y sus capacidades para investigar en grupos biológicos específicos. Por las capacidades de investigación de la isla, es seguro que existan varios registros científicos de las especies de Scolytinae y Platypodinae capturadas en ella, sin embargo, a falta de un listado, el conocimiento está disperso.

El objetivo del presente trabajo es organizar la información de las especies de Scolytinae y Platypodinae conocidas para la isla, así como enriquecer, por medio de identificaciones taxonómicas, el conocimiento de la composición faunística de ambas subfamilias de Curculionidae en la Isla Barro Colorado. Producto de este trabajo, se obtuvo un listado 
preliminar de especies de Scolytinae y Platypodinae para la isla, que podrá ser utilizado como base para estudios posteriores con este importante grupo de insectos.

\section{MATERIALES Y METODOS}

\section{Área de estudio}

La Isla Barro Colorado forma parte del Monumento Natural de Barro Colorado, ubicado en el lago Gatún del Canal de Panamá (fig. 1), en las provincias de Panamá Oeste y Colón (Leigh y Wright, 1990). La isla fue declarada reserva natural en 1923 y es administrada por el Instituto Smithsoniano desde 1946 (Leigh, 1999). Tiene un área de 1500 ha, fue creada por el aislamiento de las tierras circundante después de que el rio Chagres fuese represado en 1910 para crear el lago Gatún y completar el Canal de Panamá (Leigh y Wright, 1990). Se localiza en las coordenadas geográficas $09^{\circ} 10^{\prime} \mathrm{N}$ y $79^{\circ} 51^{\prime} \mathrm{W}$ (Windsor, 1990).

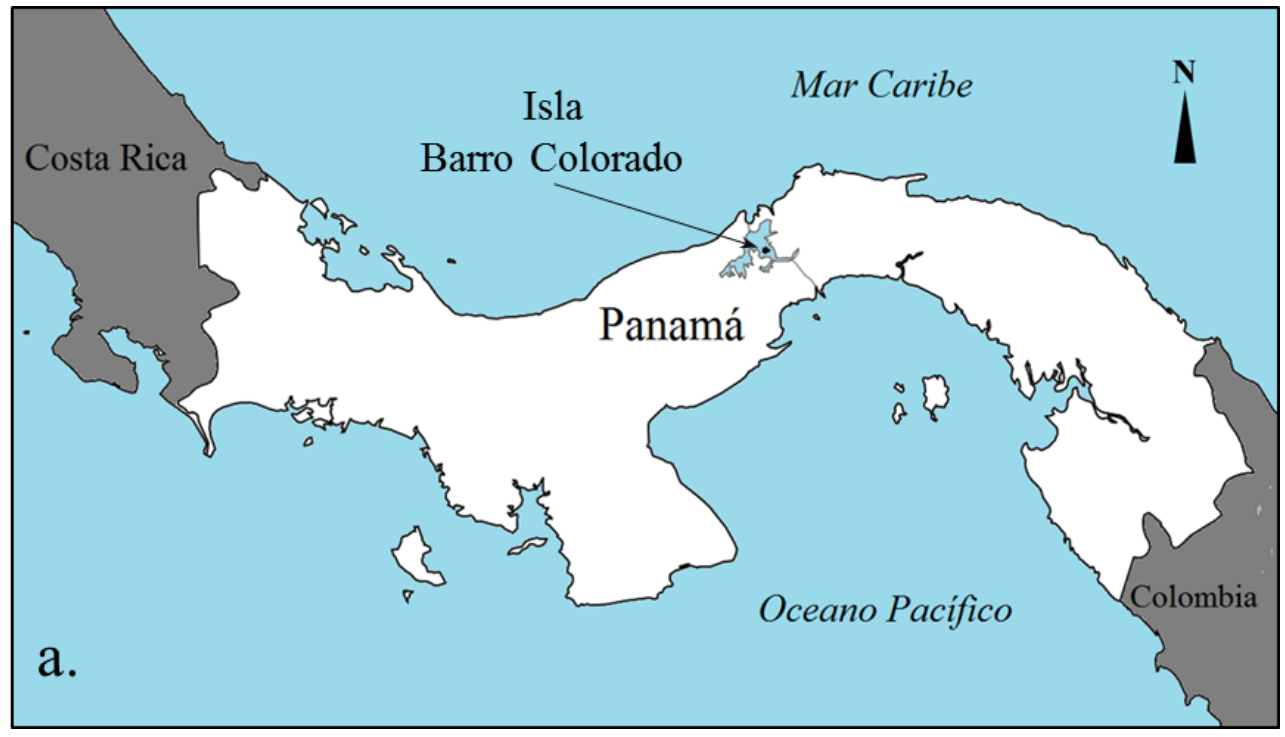



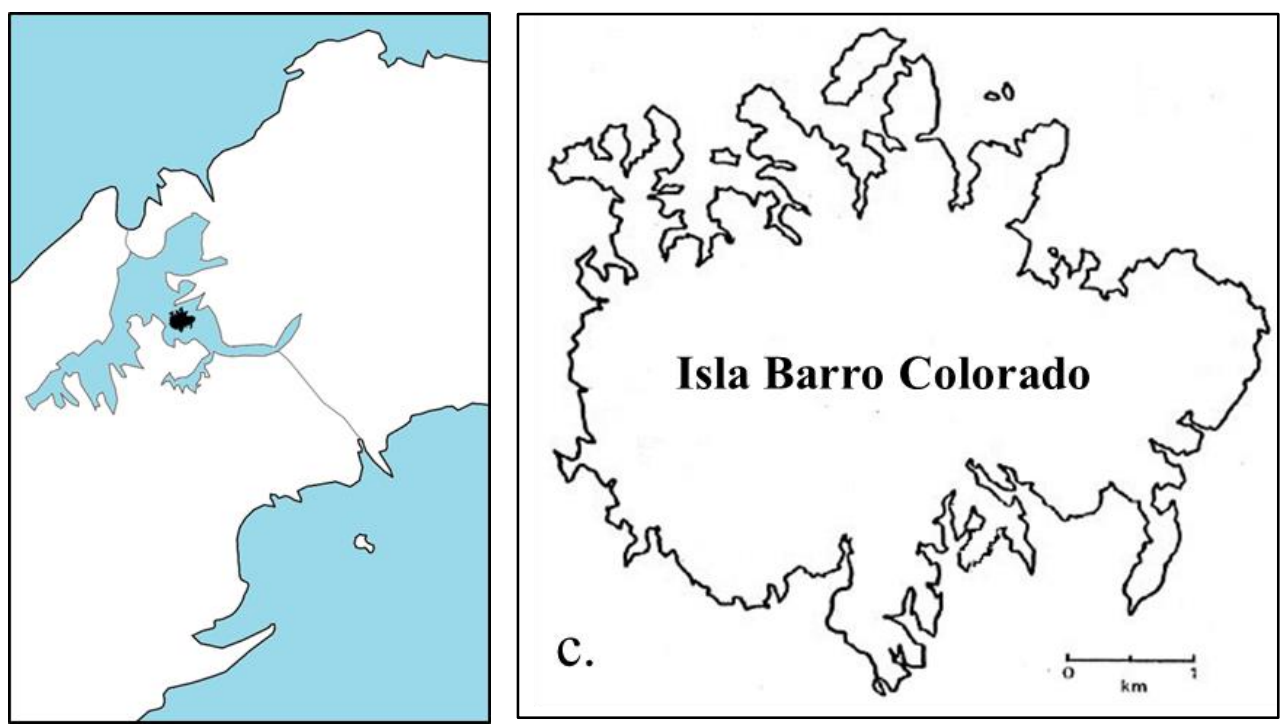

Figura 1. Ubicación de la Isla Barro Colorado. a, ubicación en la República de Panamá; b, ubicación en el Área del Canal de Panamá; c, área de la isla.

La isla presenta dos estaciones, la seca comienza a finales de diciembre o inicios de enero extendiéndose hasta finales de abril o inicios de mayo. La estación lluviosa inicia desde el mes de mayo y finaliza en diciembre (Windsor, 1990). Presenta precipitaciones hasta de 2600 milímetros anuales, temperaturas promedio anual de $27^{\circ}$, humedad relativa promedio de $83,9 \%$ y tasas de evapotranspiración de $100 \mathrm{~mm}$ por mes (Condit et al., 2000). La vegetación de la isla es semiperennifolia de bosque tropical húmedo (Knight, 1975). Registrándose 211 especies de árboles con que pueden alcanzar aturas mayores a los 10 m., de esta cantidad, cerca de 25 especies pierden sus hojas en la estación seca. Los otros árboles del dosel, del estrato medio y del sotobosque, son perennes. Las ultimas alteraciones del bosque de la isla fueron en la parte noreste realizadas durante el intento francés de construcción del canal en el siglo diecinueve (Leigh, 1999).

\section{Registro de especies}

Para conocer las especies de Scolytinae y Platypodinae reportados para la isla y adicionar nuevos registros de especies de dicha área, se realizaron tres procedimientos: 1) revisión de toda la literatura científica disponible que citara especies de Scolytinae y Platypodinae capturados en la isla, 2) revisión de especímenes de las colecciones entomológicas de la Universidad de Panamá y 3) identificación taxonómica de especímenes en muestras capturadas con trampas de luz ultravioleta en la isla por el Programa de Monitoreo de Flora y Fauna del Instituto Smithsoniano de Investigaciones (STRI).

\section{Identificación taxonómica}


Los especímenes de las subfamilias de Scolytinae y Platypodinae no identificados taxonómicamente a niveles específicos en las colecciones entomológicas y todos los especímenes en muestras fueron identificados taxonómicamente a nivel de género y especie. La determinación taxonómicamente se realizó en las instalaciones del Programa Centroamericano de Maestría en Entomología de la Universidad de Panamá (PCMENT). Debido a la abundante cantidad de especímenes, la identificación se realizó en dos etapas, la primera consistió en la creación de una colección entomológica de morfoespecies y la segunda en la identificación taxonómica de estas.

Para la identificación de Scolytinae se utilizaron las claves de Bright (1981a), Wood (1982, 1986, 2007), Wood et al., (1991a, 1991b, 1992), Smith y Cognato (2010), además se utilizaron los recursos de los sitios http://itp.lucidcentral.org (Hulcr y Smith, 2010) y http://www.barkbeetles.info (Atkinson, 2018). Para los Platypodinae se utilizaron las claves de Chapuis (1865), Blandford (1905), Schedl (1972) y Wood (1993), así como los recursos del sitio http://www.barkbeetles.info (Atkinson, 2019). Para confirmar o corroborar algunas de las identificaciones se consultó la colección de referencia del PCMENT.

\section{Organización del listado de especies}

Se realizó un listado taxonómico de especies encontradas en la isla, que integran las especies registradas en las publicaciones científicas, así como las especies identificadas en las colecciones y muestras entomológicas. En el listado de Scolytinae, la organización de los taxones superiores a género se basa fundamentalmente en Wood (1986), mientras que para Platypodinae la organización de los taxones superiores se basa en Wood (1993). Los cambios en la categoría taxonómica de los taxones superiores de ambas subfamilias, así como cambios de algunos nombres, se basan en Alonso-Zarazaga y Lyal (2009). La organización de los géneros dentro de su subtribu es alfabética, así como las especies dentro de su género.

A cada especie se le anexó información sobre el estatus de reporte para la isla, la distribución geográfica conocida, así como los hábitos alimenticios que se conocen para la especie, basados en la clasificación alimenticia de Wood (1982) para Scolytinae y la de Atkinson y Equihua-Martínez (1986a) para Platypodinae. La indicación: nuevo registro para una especie puede indicar que es una especie reportadas por primera vez para la isla o para el país, en este último caso se indica al lado del país Panamá. La abreviatura: cf., colocada en algunas especies o géneros indica que se debe comparar con la especie o género propuesto, ya que se sospecha que sea la especie o género indicado. En las especies o géneros no identificadas o que requieren ser comparados, se anexa información, en forma de nota, donde se indica la cantidad, método de captura y la ubicación física de los especímenes revisados. 


\title{
RESULTADOS Y DISCUSIÓN
}

\section{Scolytinae y Platypodinae reportados para BCI y nuevos registros}

A través de la revisión de literatura se encontraron 48 especies de Scolytinae y cinco especies de Platypodinae reportada de la Isla Barro Colorado, los registros provienen de 16 publicaciones científicas.

Por medio de la revisión del material entomológico (6577 especímenes de Scolytinae y 2415 de Platypodinae) se identificaron taxonómicamente 64 especies de Scolytinae y tres especies de Platypodinae no reportadas para la isla. De estos nuevos registros, 29 especies de Scolytinae (Chramesus minulus, C. cecropiae, Cladoctonus corumbensis, Camptocerus inoblitus, C. niger, Scolytus costellatus, Pycnarthrum brosimi, P. hispidum, P. lucidum, $P$. perditum, Micracis senta, Premnobius ambitiosus, Ambrosiodmus lecontei, A. scalaris, Coptoborus vespatorius, Dryocoetoides asperulus, Xyleborus concentus, X. geayi, X. procer, X. spathipennis, X. tumucensis, Araptus politus, Pityophthorus dimidiatus, Amphicranus argutus, A. macellus, A. spectabilis, Corthylus sobrinus, Monarthrum exornatum y M. posticum) y tres de Platypodinae (Tesserocerus retusus, Teloplatypus excisus y T. ustulatus) son nuevos registros para Panamá.

La cantidad de especies presentes en isla es mayor a la encontrada en este estudio será mayor a medida que se mejoren las identificaciones, ya que en Scolytinae 18 especies y un género requieren ser confirmadas y siente representantes de diferentes géneros no pudieron ser determinados a nivel de especie. Mientras que en Platypodinae, siente especies y un género requieren ser confirmados y cuatro representantes de diferentes géneros, tampoco pudieron ser determinados a nivel de especie.

De forma general, por medio de la revisión de literatura y material entomológico, la cantidad de especies de Scolytinae conocidos para la isla, a la fecha, llegan a 113, mientras que para Platypodinae llega a ocho. Sin embargo, la cantidad de especies de Scolytinae encontradas por medio de este estudio en Isla Barro Colorado, puede alcanzar las 138 especies. Mientras que los Platypodinae, pueden alcanzar aproximadamente 20 especies.

\section{Listado de Scolytinae y Platypodinae conocidos para BCI}

\section{Subfamilia Scolytinae}

\author{
Tribu Hylesinini \\ Subtribu Hylesinina
}


1. Phloeoborus minusculus Wood, 2007

Registro para Isla Barro Colorado: Wood (2007), Bright (2014). Distribución geográfica conocida: América: Panamá (Wood, 2007; Bright, 2014). Hábitos alimenticios: xilofagia y polifagia (Wood, 2007).

2. Phloeoborus rudis Erichson, 1836

Registro para Isla Barro Colorado: Wood (1982). Distribución geográfica conocida: América: Argentina, Belice, Bolivia, Brasil, Costa Rica, El Salvador, Guatemala, Guyana, Guyana Francesa, México, Nicaragua, Panamá, Paraguay, Perú, Venezuela (Wood, 1982; Atkinson, 1989; Wood y Bright, 1992; Bright y Skidmore, 1997; Wood, 2007). Hábitos alimenticios: xilofagia y polifagia (Wood, 1982; Wood, 2007).

3. Phloeoborus scaber Erichson, 1836

Registro para Isla Barro Colorado: Wood (1982). Distribución geográfica conocida: América: Belice, Bolivia, Brasil, México, Colombia, Costa Rica, Guatemala, Guyana Francesa, Jamaica, Nicaragua, Panamá, Perú, Trinidad y Tobago, Venezuela; Europa: Reino Unido (Wood, 1982; Atkinson y Equihua, 1986b; Wood y Bright, 1992; Wood, 2007). Hábitos alimenticios: xilofagia y polifagia (Wood, 1982; Wood, 2007).

4. Phloeoborus signatus Strohmeyer, 1909

Registro para Isla Barro Colorado: Atkinson y Equihua (1988). Distribución geográfica conocida: América: Argentina, Bolivia, Brasil, Costa Rica, Guyana Francesa, Panamá, Paraguay, Perú (Atkinson y Equihua, 1986b; Wood, 2007). Hábitos alimenticios: xilofagia y polifagia (Wood, 1982; Wood, 2007).

\section{Subtribu Phrixosomatina}

5. Phrixosoma minor Wood, 1956

Registro para Isla Barro Colorado: Wood (1982). Distribución geográfica conocida: América: Costa Rica, Honduras, Panamá (Wood, 1982). Hábitos alimenticios: fleofagia y monofagia (Wood, 1982; Wood, 2007).

6. Phrixosoma obesa Blackman, 1943

Registro para Isla Barro Colorado: nuevo registro. Distribución geográfica conocida: América: Colombia, Costa Rica, Panamá (Wood, 1982). Hábitos alimenticios: fleofagia y monofagia (Wood, 1982; Wood, 2007).

\section{Subtribu Bothrosternina}

7. Bothrosternus definitus Wood, 1968

Registro para Isla Barro Colorado: nuevo registro. Distribución geográfica conocida: América: Costa Rica, Panamá (Wood, 1982). Hábitos alimenticios: xilomicetofagia y mielofagia y polifagia (Wood, 1982).

8. Cnesinus blackmani Schedl, 1943 
Registro para Isla Barro Colorado: nuevo registro. Distribución geográfica conocida: América: Colombia, Guatemala, México, Panamá (Wood, 1982). Hábitos alimenticios: mielofagia y polifagia (Wood, 1982; Wood, 2007).

9. Cnesinus denotatus Wood, 1968

Registro para Isla Barro Colorado: Wood (1982). Distribución geográfica conocida: América: Costa Rica, Panamá, Venezuela (Wood, 1982). Hábitos alimenticios: mielofagia y polifagia (Wood, 1982; Wood, 2007).

10. Cnesinus gracilis Blandford, 1896

Registro para Isla Barro Colorado: nuevo registro. Distribución geográfica conocida: América: Belice, Brasil, Colombia, Costa Rica, Dominica, Honduras, México, Panamá, (Wood, 1982; Atkinson y Equihua, 1988; Noguera y Atkinson, 1990; Wood y Bright, 1992; Bright y Skidmore, 1997; Wood, 2007). Hábitos alimenticios: mielofagia y polifagia (Wood, 1982; Wood, 2007).

11. Cnesinus intermedius Schedl, 1936

Registro para Isla Barro Colorado: Wood (1982). Distribución geográfica conocida: América: Costa Rica, Panamá (Wood, 1982). Hábitos alimenticios: mielofagia y polifagia (Wood, 1982; Wood, 2007).

12. Cnesinus pullus Blandford, 1896

Registro para Isla Barro Colorado: nuevo registro. Distribución geográfica conocida: América: Guatemala, México, Panamá (Wood, 1982; Noguera y Atkinson, 1990). Hábitos alimenticios: mielofagia y monofagia (Wood, 1982).

13. Cnesinus setulosus Blandford, 1896

Registro para Isla Barro Colorado: nuevo registro. Distribución geográfica conocida: América: Costa Rica, Guatemala, Honduras, México, Panamá, (Wood, 1982; Bright y Skidmore, 1997). Hábitos alimenticios: mielofagia y polifagia (Wood, 1982).

\section{Cnesinus cf. C. adustus}

Nota: dos especímenes capturados en Isla Barro Colorado con trampas Pensilvania de luz ultravioleta requieren ser confirmados para esta especie. Los especímenes están depositados en la Colección del Programa Centroamericano de Maestría en Entomología de la Universidad de Panamá. Distribución geográfica conocida: Cnesinus adustus Schedl, 1948: América: Costa Rica y Honduras (Wood, 1982). Hábitos alimenticios: en especies del género Cnesinus: mielofagia y polifagia (Wood, 1982; Wood, 2007).

\section{Pagiocerus frontalis (F., 1801)}

Registro para Isla Barro Colorado: nuevo registro. Distribución geográfica conocida: América: Argentina, Bolivia, Brasil, Chile, Colombia, Cuba, El Salvador, Ecuador, Estados Unidos, Guadalupe, Guatemala, Honduras, Jamaica, México, Panamá, Perú, Trinidad y Tobago, Venezuela; Europa: Italia (Blandford, 1905; Wood, 1982; Atkinson y Equihua,1988; Wood y Bright, 1992; Bright y Skidmore, 1997; Wood, 2007). Hábitos alimenticios: espermatofagia y monofagia (Wood, 1982). 


\section{Subtribu Phloeotribina}

16. Phloeotribus demessus Blandford, 1897

Registro para Isla Barro Colorado: Wood (1982). Distribución geográfica conocida: América: Costa Rica, México, Panamá (Wood, 1982). Hábitos alimenticios: fleofagia y oligofagia (Wood, 1982).

17. Phloeotribus simplex Wood, 1967

Registro para Isla Barro Colorado: Wood (1967), Wood (1982). Distribución geográfica conocida: América: Panamá (Wood, 1982). Hábitos alimenticios: fleofagia y monofagia (Wood, 1982).

\section{Subtribu Phloeosinina}

18. Chramesus cecropiae Wood, 1969

Registro para Isla Barro Colorado: nuevo registro. Distribución geográfica conocida: América: Costa Rica, Panamá (nuevo registro) (Wood, 1982). Hábitos alimenticios: fleofagia y monofagia (Wood, 1982).

19. Chramesus minulus Wood, 1969

Registro para Isla Barro Colorado: nuevo registro. Distribución geográfica conocida: América: Honduras, México, Panamá (nuevo registro) (Wood, 1982; Atkinson, y Equihua, 1986). Hábitos alimenticios: fleofagia y monofagia (Wood, 1982).

20. Cladoctonus corumbensis Eggers, 1951

Registro para Isla Barro Colorado: nuevo registro. Distribución geográfica conocida: América: Bolivia, Brasil, Colombia, Panamá (nuevo registro) (Wood, 1971; Wood, 1985). Hábitos alimenticios: fleofagia, solo un hospedero conocido (Wood, 2007).

\section{Tribu Scolytini Subtribu Scolytina}

21. Camptocerus aeneipennis (F., 1801)

Registro para Isla Barro Colorado: Smith y Cognato (2010). Distribución geográfica conocida: América: Argentina, Bolivia, Brasil, Colombia, Costa Rica, Ecuador, Guyana Francesa, Guyana, Panamá, Perú, Surinam, Venezuela (Smith y Cognato, 2010). Hábitos alimenticios: xilomicetofagia, posiblemente oligofagia (Wood, 2007; Atkinson y Rabaglia, 2010; Smith y Cognato, 2010).

22. Camptocerus auricomus Blandford, 1896

Registro para Isla Barro Colorado: nuevo registro. Distribución geográfica conocida: América: Costa Rica, Ecuador, Guatemala, Guyana, México, Nicaragua, Panamá, Trinidad y Tobago, Venezuela (Wood, 1982; Wood y Bright, 1992; Smith y Cognato, 2010). Hábitos alimenticios: xilomicetofagia y oligofagia (Wood, 1982; Atkinson y Rabaglia, 2010). 
23. Camptocerus inoblitus (Schedl, 1939)

Registro para Isla Barro Colorado: nuevo registro. Distribución geográfica conocida: América: Argentina, Brasil, Panamá (nuevo registro) (Smith y Cognato, 2010). Hábitos alimenticios: xilomicetofagia (Wood, 2007).

24. Camptocerus niger (F., 1801)

Registro para Isla Barro Colorado: nuevo registro. Distribución geográfica conocida: América: Brasil, Colombia, Costa Rica, Guyana, Guyana Francesa, Panamá (nuevo registro), Perú, Surinam, Venezuela; Europa: Reino Unido (Wood, 1982; Smith y Cognato, 2010). Hábitos alimenticios: xilomicetofagia y oligofagia (Wood, 1982).

25. Cnemonyx exiguus (Blandford, 1896)

Registro para Isla Barro Colorado: nuevo registro. Distribución geográfica conocida: América: Costa Rica, Panamá (Wood, 1982). Hábitos alimenticios: fleofagia y monofagia (Wood, 1982).

26. Loganius vagabundus Wood, 1961

Registro para Isla Barro Colorado: Wood (1982). Distribución geográfica conocida: América: Antigua, Estados Unidos, Panamá, Puerto Rico, República Dominicana (Wood, 1982; Bright, 1981a). Hábitos alimenticios: fleofagia y monofagia (Wood, 1982).

27. Scolytus costellatus Chapuis, 1869

Registro para Isla Barro Colorado: nuevo registro. Distribución geográfica conocida: América: Brasil, Costa Rica, Panamá (nuevo registro), Venezuela (Wood, 2007). Hábitos alimenticios: fleofagia y monofagia (Wood, 1982).

\section{Subtribu Hexacolina}

28. Gymnochilus reitteri Eichhoff, 1878

Registro para Isla Barro Colorado: Wood (1982). Distribución geográfica conocida: América: Costa Rica, Guatemala, México, Nicaragua, Panamá, Trinidad y Tobago (Wood, 1982; Bright y Skidmore, 2002). Hábitos alimenticios: fleofagia y monofagia (Wood, 1982).

29. Microborus boops Blandford, 1897

Registro para Isla Barro Colorado: Wood (1982). Distribución geográfica conocida: África: Camerún, Costa de Marfil, Madagascar, República Democrática del Congo, Uganda; América: Costa Rica, Guatemala, Honduras, Jamaica, Panamá, Trinidad y Tobago (Wood, 1982; Bright y Skidmore, 1997). Hábitos alimenticios: fleofagia, un solo hospedero reportado (Wood, 1982).

30. Pycnarthrum brosimi Wood, 1971

Registro para Isla Barro Colorado: nuevo registro. Distribución geográfica conocida: América: Colombia, Costa Rica, Nicaragua, Panamá (nuevo registro), Venezuela (Wood, 1982; Atkinson y Equihua, 1988). Hábitos alimenticios: fleofagia y monofagia (Wood, 2007). 
31. Pycnarthrum hispidum (Ferrari, 1867)

Registro para Isla Barro Colorado: nuevo registro. Distribución geográfica conocida: América: Bahamas, Brasil, Costa Rica, Cuba, Estados Unidos, El Salvador, Guadalupe, Guatemala, Guyana, México, República Dominicana, Panamá (nuevo registro), Puerto Rico, Trinidad y Tobago, Venezuela (Wood, 1982; Bright y Skidmore, 1997; Peck y Thomas, 1998; Bright y Torres, 2006; Wood, 2007). Hábitos alimenticios: fleofagia y monofagia (Wood, 2007).

32. Pycnarthrum lucidum Wood, 1971

Registro para Isla Barro Colorado: nuevo registro. Distribución geográfica conocida: América: Costa Rica, Panamá (nuevo registro) (Wood, 1982). Hábitos alimenticios: fleofagia y monofagia (Wood, 2007).

33. Pycnarthrum pallidum (Chapuis, 1869)

Registro para Isla Barro Colorado: Atkinson y Equihua (1988). Distribución geográfica conocida: América: Antillas Menores, Barbados, Brasil, Guadalupe, Guatemala, Honduras, Jamaica, México, Panamá, Venezuela (Wood, 1982; Atkinson y Equihua, 1988; Wood y Bright, 1992; Bright y Skidmore, 1997; Wood, 2007; Peck, 2009). Hábitos alimenticios: fleofagia y oligofagia (Wood, 2007).

34. Pycnarthrum perditum Wood, 1971

Registro para Isla Barro Colorado: nuevo registro. Distribución geográfica conocida: América: Honduras, Panamá (nuevo registro) (Wood, 1982). Hábitos alimenticios: fleofagia, no se conoce hospederos (Wood, 2007).

35. Scolytodes acares Wood, 1969

Registro para Isla Barro Colorado: Wood (1982). Distribución geográfica conocida: América: Costa Rica, Panamá (Wood, 1982). Hábitos alimenticios: fleofagia y monofagia (Wood, 1982).

36. Scolytodes crassus Wood, 1971

Registro para Isla Barro Colorado: Wood (1982). Distribución geográfica conocida: América: Panamá (Wood, 1982). Hábitos alimenticios: fleofagia y monofagia (Wood, 1982).

37. Scolytodes nanellus Wood, 1967

Registro para Isla Barro Colorado: Wood (1982), Jordal (1998b). Distribución geográfica conocida: América: Panamá (Wood, 1982; Jordal 1998b). Hábitos alimenticios: fleofagia y monofagia (Wood, 1982).

38. Scolytodes obesus Wood, 1975

Registro para Isla Barro Colorado: Wood (1975), Wood (1982). Distribución geográfica conocida: América: Panamá (Wood, 1982). Hábitos alimenticios: fleofagia y monofagia (Wood, 1982).

39. Scolytodes suspectus Jordal, 1998 
Registro para Isla Barro Colorado: Jordal, (1998a). Distribución geográfica conocida: América: Panamá (Jordal, 1998a). Hábitos alimenticios: según Jordal (1998a) posiblemente similar a otras especies del género: fleofagia y monofagia.

40. Scolytodes cf. S. perditus

Nota: un espécimen capturado en Isla Barro Colorado con trampas Pensilvania de luz ultravioleta requiere ser confirmado para esta especie. El espécimen está depositado en la colección del Programa Centroamericano de Maestría en Entomología de la Universidad de Panamá. Distribución geográfica conocida: Scolytodes perditus Wood, 1967: América: Panamá (Wood, 1982). Hábitos alimenticios: en las especies del género Scolytodes: fleofagia y monofagia (Wood, 1982).

\section{Subtribu Micracidina}

41. Micracis senta Wood, 1971

Registro para Isla Barro Colorado: nuevo registro. Distribución geográfica conocida: América: Panamá (nuevo registro), Venezuela (Wood, 1982). Hábitos alimenticios: xilofagia, hospederos no conocidos (Wood, 2007).

\section{Subtribu Ipina}

42. Acanthotomicus mimicus (Schedl, 1961)

Registro para Isla Barro Colorado: Wood (1982). Distribución geográfica conocida: América: Brasil, Costa Rica, Jamaica, México, Panamá, República Dominicana, Venezuela (Wood, 1982; Wood, 2007; Salgado, 2008). Hábitos alimenticios: fleofagia y monofagia (Wood, 1982).

43. Premnobius ambitiosus (Schaufuss, 1897)

Registro para Isla Barro Colorado: nuevo registro. Distribución geográfica conocida: África: Camerún, Congo, Costa de Marfil, Gabón, Ghana, Guinea, Guinea Ecuatorial, Liberia, Nigeria, Sierra Leona, Tanzania; América: Bolivia, Brasil, Colombia, Panamá (nuevo registro), Venezuela; Europa: Reino Unido (Wood, 1982; Wood y Bright, 1992; Wood, 2007). Hábitos alimenticios: xilomicetofagia y polifagia (Wood, 1982).

44. Premnobius cavipennis Eichhoff, 1878

Registro para Isla Barro Colorado: nuevo registro. Distribución geográfica conocida: África: Angola, Burundi, Congo, Costa de Marfil, Etiopia, Gabón, Ghana, Guinea Ecuatorial, Kenia, Madagascar, Malawi, Mauritania, Mozambique, Namibia, Nigeria, Ruanda, Somalia, Tanzania, Uganda, Zambia, Zimbabue; América: Belice, Brasil, Costa Rica, Colombia, Cuba, Bahamas, Dominica, Estados Unidos, Guyana Francesa, Honduras, México, Panamá, Puerto Rico, Surinam, Trinidad y Tobago, Venezuela; Europa: Reino Unido (Wood, 1982; Atkinson y Equihua, 1988; Wood y Bright, 1992; Bright y Skidmore, 1997; Bright y Skidmore, 2002; Guerra, 2004; Bright y Torres, 2006; Salgado, 2008). Hábitos alimenticios: xilomicetofagia y polifagia (Wood, 1982). 


\section{Subtribu Dryocoetina}

45. Coccotrypes aciculatus Schedl, 1952

Registro para Isla Barro Colorado: Wood (1982). Distribución geográfica conocida: América: Brasil, Costa Rica, Panamá; Oceanía: Nueva Guinea (Wood, 1982). Hábitos alimenticios: espermatofagia y polifagia (Wood, 1982).

46. Coccotrypes cyperi (Beeson, 1929)

Registro para Isla Barro Colorado: Wood (1982). Distribución geográfica conocida: América: Brasil, Costa Rica, Estados Unidos, Guadalupe, Honduras, México, Panamá, Puerto Rico, República Dominicana, Surinam; Asia: Birmania, China, India, Indonesia, Malasia, Sri Lanka, Vietnam; Europa: Martinica; Oceanía: Australia, Fiyi, Islas Cook, Micronesia, Samoa, Tahití, Tonga (Wood, 1982; Wood y Bright, 1992; Bright y Skidmore, 1997; Bright y Skidmore, 2002; Guerra, 2004; Bright y Torres, 2006; Salgado, 2008). Hábitos alimenticios: espermatofagia fleofagia y polifagia (Wood, 1982).

\section{Subtribu Xyleborina}

47. Ambrosiodmus lecontei Hopkins, 1915

Registro para Isla Barro Colorado: nuevo registro. Distribución geográfica conocida: América: Brasil, Cuba, Estados Unidos, Jamaica, Panamá (nuevo registro), Puerto Rico, República Dominicana (Wood, 1982; Bright y Skidmore, 1997; Bright y Torres, 2006). Hábitos alimenticios: xilomicetofagia y polifagia (Wood, 1982).

48. Ambrosiodmus scalaris (Schedl, 1942)

Registro para Isla Barro Colorado: nuevo registro. Distribución geográfica conocida: América: Costa Rica, México, Panamá (nuevo registro), Surinam (Wood, 1982; Wood, 2007). Hábitos alimenticios: xilomicetofagia y polifagia (Wood, 1982).

49. Coptoborus vespatorius (Schedl, 1931)

Registro para Isla Barro Colorado: nuevo registro. Distribución geográfica conocida: América: Argentina, Brasil, Colombia, Costa Rica, Guatemala, México, Panamá (nuevo registro), Trinidad y Tobago, Venezuela (Hopkins, 1915; Wood, 1982; Wood, 2007). Hábitos alimenticios: xilomicetofagia y polifagia (Wood, 1982).

50. Coptoborus cf. C. cuneatus

Nota: un espécimen capturado en Isla Barro Colorado con trampa Pensilvania de luz ultravioleta requieren ser confirmados para esta especie. El espécimen está en la Colección del Programa Centroamericano de Maestría en Entomología de la Universidad de Panamá. Distribución geográfica conocida: Coptoborus cuneatus (Eichhoff, 1878): América: Panamá, Perú, Venezuela (Wood, 1982). Hábitos alimenticios: en C. cuneatus: xilomicetofagia (Wood, 1982).

\section{Coptoborus cf. C. pseudotenuis}

Nota: un espécimen capturado en Isla Barro Colorado con trampa Pensilvania de luz ultravioleta requiere ser confirmado para esta especie. El espécimen está en la Colección 
del Programa Centroamericano de Maestría en Entomología de la Universidad de Panamá. Distribución geográfica conocida: Coptoborus pseudotenuis (Schedl, 1936): América: Brasil, Costa Rica, Estados Unidos, México, Panamá, Trinidad y Tobago, Venezuela (Wood, 1982; Bright y Skidmore, 2002; Guerra, 2004; Salgado, 2008; Atkinson et al., 2009). Hábitos alimenticios: en C. pseudotenuis: xilomicetofagia y polifagia (Wood, 1982).

\section{Coptoborus cf. C. tolimanus}

Nota: un espécimen capturado en Isla Barro Colorado con trampa Pensilvania de luz ultravioleta requiere ser confirmado para esta especie. El espécimen está en la Colección del Programa Centroamericano de Maestría en Entomología de la Universidad de Panamá. Distribución geográfica conocida: Coptoborus tolimanus (Eggers, 1928): América: Brasil, Colombia, Costa Rica, México, Panamá, Venezuela; Europa: Reino Unido (Wood, 1982; Wood y Bright, 1992; Guerra, 2004, Salgado, 2008; Pérez De La Cruz et al., 2009b). Hábitos alimenticios: en C. tolimanus: xilomicetofagia y polifagia (Wood, 1982).

53. Dryocoetoides asperulus (Eggers, 1931)

Registro para Isla Barro Colorado: nuevo registro. Distribución geográfica conocida: América: Brasil, Costa Rica, México, Panamá (nuevo registro) (Wood, 1982; Wood y Bright, 1992). Hábitos alimenticios: xilomicetofagia y polifagia (Wood, 1982).

54. Dryocoetoides capucinus (Eichhoff, 1869)

Registro para Isla Barro Colorado: nuevo registro. Distribución geográfica conocida: América: Brasil, Colombia, Costa Rica, Guadalupe, Guatemala, Guyana, Jamaica, México, Panamá, República Dominicana, Venezuela (Bright, 1981b; Wood, 1982; Wood y Bright, 1992). Hábitos alimenticios: xilomicetofagia y polifagia (Wood, 1982).

\section{Dryocoetoides cf. D. velutinus}

Nota: 12 especímenes capturados en Isla Barro Colorado con trampa Pensilvania de luz ultravioleta requieren ser confirmados para esta especie. Los especímenes están en la Colección del Programa Centroamericano de Maestría en Entomología de la Universidad de Panamá. Distribución geográfica conocida: Dryocoetoides velutinus Wood, 1974: América: Venezuela (Wood y Bright, 1992). Hábitos alimenticios: especies del género Dryocoetoides: xilomicetofagia (Wood, 2007).

56. Euwallacea fornicatus (Eichhoff, 1868)

Registro para Isla Barro Colorado: nuevo registro. Distribución geográfica conocida: África: Comoras, Reunión; América: Brasil, Costa Rica, Estados Unidos, Panamá; Asia: Bangladesh, Birmania, China, Filipinas, India, Indonesia, Japón, Malasia, Sri Lanka, Vietnam; Europa: Reino Unido. Oceanía: Australia, Islas Carolinas, Fiyi, Nueva Bretaña, Nueva Zelanda, Samoa (Wood, 1982; Wood y Bright, 1992; Kirkendall y Ødegaard, 2007; Wood, 2007; Salgado, 2008). Hábitos alimenticios: xilomicetofagia y polifagia (Wood, 2007).

57. Euwallacea sp.

Nota: tres especímenes capturados en Isla Barro Colorado por medio de trampa Pensilvania de luz ultravioleta fueron organizados en dos morfoespecies diferentes a $E$. 
fornicatus. Los especímenes están en la Colección del Programa Centroamericano de Maestría en Entomología de la Universidad de Panamá. Distribución geográfica conocida: género Euwallacea en América está representado por especies introducidas (Wood 2007). Sus especies se distribuyen en Asia y Australia (Wood y Bright, 1992). Hábitos alimenticios: en las especies del género Euwallacea: xilomicetofagia y polifagia (Wood, 2007).

58. Sampsonius dampfi Schedl, 1940

Registro para Isla Barro Colorado: Wood (1982). Distribución geográfica conocida: América: Brasil, Colombia, Costa Rica, Honduras, México, Panamá, Perú, Trinidad y Tobago, Venezuela (Wood, 1982; Wood y Bright, 1992; Guerra, 2004; Wood, 2007; Petrov y Mandelshtam, 2009). Hábitos alimenticios: xilomicetofagia y polifagia (Wood, 1982).

59. Sampsonius detractus Wood, 1974

Registro para Isla Barro Colorado: nuevo registro. Distribución geográfica conocida: América: Brasil, Panamá (Wood, 1982; Wood y Bright, 1992). Hábitos alimenticios: xilomicetofagia y polifagia (Wood, 1982).

60. Taurodemus flavipes (F., 1801)

Registro para Isla Barro Colorado: Wood (1982). Distribución geográfica conocida: América: Brasil, Colombia, Costa Rica, Guatemala, México, Panamá, Venezuela; Europa: Reino Unido (Wood, 1982; Atkinson, y Equihua, 1986; Wood y Bright, 1992). Hábitos alimenticios: xilomicetofagia y polifagia (Wood, 1982).

61. Taurodemus sharpi (Blandford, 1898)

Registro para Isla Barro Colorado: Wood (1982). Distribución geográfica conocida: América: Costa Rica, Guatemala, Honduras, México y Nicaragua, Panamá (Wood, 1974; Wood, 1982; Atkinson, 2018; Rodríguez-Flores, 2019). Hábitos alimenticios: xilomicetofagia y polifagia (Wood, 1982).

62. Theoborus coartatus (Sampson, 1921)

Registro para Isla Barro Colorado: nuevo registro. Distribución geográfica conocida: América: Brasil, Colombia, Costa Rica, Jamaica, México, Panamá, Trinidad y Tobago (Wood, 1982; Wood y Bright, 1992). Hábitos alimenticios: xilomicetofagia y polifagia (Wood, 1982).

63. Theoborus crinitulus (Wood, 1974)

Registro para Isla Barro Colorado: Wood (1974), Wood y Bright (1992). Distribución geográfica conocida: América: Panamá, Venezuela (Wood y Bright, 1992; Wood, 2007). Hábitos alimenticios: xilomicetofagia y polifagia (Wood, 1982).

64. Theoborus molestulus (Wood, 1975)

Registro para Isla Barro Colorado: Wood (1982). Distribución geográfica conocida: América: Panamá (Wood, 1982). Hábitos alimenticios: xilomicetofagia y polifagia (Wood, 1982). 
65. Theoborus theobromae Hopkins, 1915

Registro para Isla Barro Colorado: Wood (1982). Distribución geográfica conocida: América: Barbados, Colombia, Costa Rica, Dominica, Guyana Francesa, México, Panamá, San Vicente y Las Granadinas, Trinidad y Tobago, Venezuela (Hopkins, 1915; Wood, 1982). Hábitos alimenticios: xilomicetofagia (Wood, 1982).

66. Xyleborus affinis Eichhoff, 1868

Registro para Isla Barro Colorado: nuevo registro. Distribución geográfica conocida: África: Angola, Azores, Birmania, Camerún, Congo, Costa de Marfil, Guinea Ecuatorial, Etiopía, Gabón, Guinea, Kenia, Liberia, Madagascar, Malawi, Mauritania, Mauricio, Nigeria, Reunión, Ruanda, Senegal, Seychelles, Sierra Leona, Tanzania, Uganda, Zambia; América: Barbados, Belice, Brasil, Canadá, Chile, Colombia, Costa Rica, Cuba, Ecuador, El Salvador, Estados Unidos, Guadalupe, Jamaica, México, Panamá, Puerto Rico, República Dominicana, San Vicente y Las Granadinas, Surinam, Trinidad y Tobago, Venezuela; Asia: India, Indonesia, Israel, Malasia, Sri Lanka; Europa: Reino Unido; Oceanía: Islas Cook, Fiyi, Islas Marianas, Palau, Samoa, Tahití (Hopkins, 1915; Bright, 1982; Wood, 1982; Estrada y Atkinson, 1989; Wood y Bright, 1992; Bright y Skidmore, 2002; Guerra, 2004; Bright y Torres, 2006; Wood, 2007; Douglas et. al. 2013). Hábitos alimenticios: xilomicetofagia y polifagia (Wood, 1982).

67. Xyleborus asper Eggers, 1933

Registro para Isla Barro Colorado: Wood (1982). Distribución geográfica conocida: América: Brasil, Colombia, Costa Rica, Guyana Francesa, Panamá, Perú, Trinidad y Tobago, Venezuela (Wood, 1982; Bright y Skidmore, 2002). Hábitos alimenticios: xilomicetofagia y polifagia (Wood, 1982).

68. Xyleborus caraibicus Eggers, 1941

Registro para Isla Barro Colorado: Wood (1982). Distribución geográfica conocida: América: Bolivia, Brasil, Colombia, Costa Rica, Dominica, Guadalupe, Panamá, Trinidad y Tobago, Venezuela (Bright, 1982; Wood, 1982; Wood y Bright, 1992; Wood, 2007). Hábitos alimenticios: xilomicetofagia y polifagia (Wood, 1982).

69. Xyleborus concentus Wood, 1974

Registro para Isla Barro Colorado: nuevo registro. Distribución geográfica conocida: América: Costa Rica, Ecuador, Panamá (nuevo registro), Venezuela (Wood, 1974; Bright y Skidmore, 2002). Hábitos alimenticios: xilomicetofagia y polifagia (Wood, 1982).

70. Xyleborus discretus Eggers, 1933

Registro para Isla Barro Colorado: Bright y Skidmore (2002). Distribución geográfica conocida: América: Costa Rica, Guyana, Guyana Francesa, México, Panamá, Perú, Venezuela (Wood, 1982; Bright y Skidmore, 2002). Hábitos alimenticios: xilomicetofagia y polifagia (Wood, 1982).

71. Xyleborus ferox Blandford, 1898 
Registro para Isla Barro Colorado: nuevo registro. Distribución geográfica conocida: América: Colombia, Costa Rica, Panamá (Wood, 1982; Salgado, 2008). Hábitos alimenticios: xilomicetofagia y polifagia (Wood, 1982).

72. Xyleborus ferrugineus (F., 1801)

Registro para Isla Barro Colorado: nuevo registro. Distribución geográfica conocida: África: Angola, Azores, Botsuana, Burkina Faso, Birmania, Cabo Verde, Camerún, Costa de Marfil, Etiopía, Gabón, Ghana, Guinea Ecuatorial, Kenia, Liberia, Madagascar, Malawi, Mauritania, Reunión, Ruanda, Senegal, Seychelles, Sierra Leona, Somalia, Sudan, Tanzania, Togo, Zimbabue; América: Argentina, Bolivia, Brasil, Canadá, Colombia, Costa Rica, Cuba, Dominica, Ecuador, El Salvador, Estados Unidos, Guadalupe, Guatemala, México, Panamá, Puerto Rico, Trinidad y Tobago, Uruguay, Venezuela; Europa: Reino Unido (Wood, 1961; Wood, 1982; Atkinson, y Equihua, 1985; Wood y Bright, 1992; Bright y Skidmore, 1997; Bright y Skidmore, 2002; Guerra, 2004; Wood, 2007; Salgado, 2008). Hábitos alimenticios: xilomicetofagia y polifagia (Wood, 1982).

73. Xyleborus geayi Hagedorn, 1905

Registro para Isla Barro Colorado: nuevo registro. Distribución geográfica conocida: América: Brasil, Colombia, Costa Rica, Guyana Francesa, Panamá (nuevo registro), Venezuela (Wood, 1982). Hábitos alimenticios: xilomicetofagia y polifagia (Wood, 1982).

74. Xyleborus horridatus Wood, 1967

Registro para Isla Barro Colorado: Wood (1967). Distribución geográfica conocida: América: Colombia, Costa Rica, Honduras, Panamá (Wood, 1967; Atkinson y Equihua, 1988, Guerra, 2004). Hábitos alimenticios: xilomicetofagia y polifagia (Wood, 1982).

75. Xyleborus macer Blandford, 1898

Registro para Isla Barro Colorado: nuevo registro. Distribución geográfica conocida: América: Belice, Brasil, Colombia, Costa Rica, Guatemala, México, Nicaragua, Panamá, Puerto Rico, Venezuela; Europa: Reino Unido (Bright, 1981b; Wood, 1982; Atkinson y Equihua, 1988; Wood y Bright, 1992). Hábitos alimenticios: xilomicetofagia y polifagia (Wood, 1982).

76. Xyleborus posticus Eichhoff, 1869

Registro para Isla Barro Colorado: Wood (1982). Distribución geográfica conocida: América: Brasil, Belice, Colombia, Costa Rica, Guadalupe, Guatemala, Guyana, Honduras, México, Panamá, Trinidad y Tobago, Venezuela (Wood, 1961; Wood, 1982; Atkinson y Equihua, 1988; Wood y Bright, 1992; Guerra, 2004; Wood, 2007). Hábitos alimenticios: xilomicetofagia (Wood, 1982).

77. Xyleborus princeps Blandford, 1898

Registro para Isla Barro Colorado: nuevo registro. Distribución geográfica conocida: América: Colombia, Costa Rica, Ecuador, Nicaragua, Panamá (Wood, 1961; Wood 1982). Hábitos alimenticios: xilomicetofagia y polifagia (Wood, 1982). 
78. Xyleborus procer Eichhoff, 1878

Registro para Isla Barro Colorado: nuevo registro. Distribución geográfica conocida: América: Brasil, Colombia, Guatemala, Guyana Francesa, Panamá (nuevo registro), Surinam (Wood, 1982; Wood y Bright, 1992; Wood, 2007). Hábitos alimenticios: xilomicetofagia y polifagia (Wood, 1982).

79. Xyleborus spathipennis Eichhoff, 1868

Registro para Isla Barro Colorado: nuevo registro. Distribución geográfica conocida: América: Brasil, Colombia, Costa Rica, Ecuador, México, Nicaragua, Panamá (nuevo registro), Perú, Surinam (Wood, 1982; Wood y Bright, 1992). Hábitos alimenticios: xilomicetofagia y polifagia (Wood, 1982).

80. Xyleborus spinulosus Blandford, 1898

Registro para Isla Barro Colorado: nuevo registro. Distribución geográfica conocida: América: Argentina, Brasil, Colombia, Costa Rica, Guatemala, Ecuador, Estados Unidos, Haití, Honduras, México, Panamá, Perú, República Dominicana, Venezuela (Wood, 1982; Wood y Bright, 1992; Bright y Skidmore, 2002; Wood, 2007; Salgado, 2008; Atkinson y Riley, 2013). Hábitos alimenticios: xilomicetofagia y polifagia (Wood, 1982).

81. Xyleborus tumucensis Hagedorn, 1905

Registro para Isla Barro Colorado: nuevo registro. Distribución geográfica conocida: América: Guyana Francesa, Panamá (nuevo registro) (Wood, 1982). Hábitos alimenticios: xilomicetofagia y polifagia (Wood, 1982).

82. Xyleborus volvulus (F., 1775)

Registro para Isla Barro Colorado: nuevo registro. Distribución geográfica conocida: África: Angola, Burundi, Camerún, Costa de Marfil, Etiopía, Gabón, Guinea, Guinea Ecuatorial, Kenia, Madagascar, Mauricio, Mozambique, Namibia, Nigeria, Ruanda, Seychelles, Sierra Leona, Somalia, Sudan, Tanzania, Uganda, Zaire, Zimbabue; América: Brasil, Colombia, Costa Rica, Cuba, Ecuador, El Salvador, Estados Unidos, Granada, México, Panamá. Puerto Rico, República Dominicana, Trinidad y Tobago, Uruguay; Asia: Birmania, China, Corea, Filipinas, Indonesia, Japón, Malasia, Tailandia; Europa: Reino Unido. Oceanía: Australia, Guadalcanal, Samoa, Samoa Americana (Hopkins, 1915; Wood, 1982; Wood y Bright, 1992; Bright y Skidmore, 1997; Guerra, 2004; Bright y Torres, 2006). Hábitos alimenticios: xilomicetofagia y polifagia (Wood, 1982).

\section{Xyleborus sp.}

Nota: un espécimen capturados en Isla Barro Colorado por medio de trampa Pensilvania de luz ultravioleta. El espécimen está en la Colección del Programa Centroamericano de Maestría en Entomología de la Universidad de Panamá. Distribución geográfica conocida: El género Xyleborus tiene una distribución mundial. 73 especies ocurren en Norte y Centroamérica (Wood, 1982) y 78 en Suramérica (Wood 2007). Hábitos alimenticios: en las especies del género Xyleborus: xilomicetofagia y polifagia (Wood, 2007).

84. Xyleborinus bicornatulus (Wood, 1967) 
Registro para Isla Barro Colorado: nuevo registro. Distribución geográfica conocida: América: Colombia, Costa Rica, Panamá (Wood, 1967; Guerra, 2004). Hábitos alimenticios: xilomicetofagia y polifagia (Wood, 1982).

85. Xyleborinus exiguus (Walker, 1859)

Registro para Isla Barro Colorado: nuevo registro. Distribución geográfica conocida: América: Costa Rica, Panamá; Asia: Birmania, Filipinas, India, Indonesia, Malasia, Nepal, Sri Lanka, Tailandia, Vietnam; Europa: Reino Unido. Oceanía: Fiyi, Guam, Islas Marianas, Islas Salomón, Islas Sociedad, Nueva Caledonia, Palaos, Papúa Nueva Guinea (Wood y Bright, 1992; Guerra, 2004; Kirkendall y Ødegaard, 2007). Hábitos alimenticios: xilomicetofagia y polifagia (Wood, 1982).

86. Xyleborinus gracilis (Eichhoff, 1868)

Registro para Isla Barro Colorado: nuevo registro. Distribución geográfica conocida: África: Azores. América: Argentina, Brasil, Colombia, Costa Rica, Ecuador, Estados Unidos, Guadalupe, México, Panamá, Puerto Rico, Venezuela (Wood, 1982; Estrada y Atkinson, 1989: Bright y Skidmore, 2002; Guerra, 2004; Wood, 2007; Salgado, 2008). Hábitos alimenticios: xilomicetofagia y polifagia (Wood, 1982).

87. Xyleborinus intersetosus (Blandford, 1898)

Registro para Isla Barro Colorado: Wood (1982). Distribución geográfica conocida: América: Brasil, Colombia, Costa Rica, Ecuador, Guadalupe, Guatemala, Guyana, México, Panamá, Surinam, Venezuela (Wood, 1982; Wood y Bright, 1992; Guerra, 2004; Wood, 2007). Hábitos alimenticios: xilomicetofagia y polifagia (Wood, 1982).

88. Xylosandrus crassiusculus (Motschulsky, 1866)

Registro para Isla Barro Colorado: nuevo registro. Distribución geográfica conocida: África: Camerún, Costa de Marfil, Guinea Ecuatorial, Ghana, Kenia, Madagascar, Mauritania, Mauricio, Nigeria, Seychelles, Sierra Leona, Tanzania, Zaire; América: Brasil, Canadá, Costa Rica, Estados Unidos, Guyana Francesa, Panamá; Asia: Birmania, Bután, China, Filipinas, India, Indonesia, Japón, Corea, Malasia, Sri Lanka, Vietnam; Europa: Alemania, Reino Unido. Oceanía: Fiyi, Nueva Caledonia, Nueva Zelanda, Palaos (Wood, 1982; Wood y Bright, 1992; Kirkendall y Ødegaard, 2007; Douglas et al., 2013; Flechtmann y Atkinson, 2016). Hábitos alimenticios: xilomicetofagia y polifagia (Wood, 1982).

89. Xylosandrus morigerus (Blandford, 1894)

Registro para Isla Barro Colorado: Wood (1982). Distribución geográfica conocida: África: Madagascar; América: Brasil, Colombia, Costa Rica, Ecuador, Honduras, México, Panamá, Puerto Rico, Venezuela. Asia: China, Filipinas, India, Indonesia, Jordania, Líbano, Malasia, Mauricio, Sri Lanka, Vietnam, Zaire. Europa: Inglaterra, Francia, Italia, Reino Unido, Checoslovaquia. Oceanía: Australia, Islas Carolinas, Fiyi, Islas Marianas, Papúa Nueva Guinea, Samoa (Wood, 1961; Wood, 1982; Wood y Bright, 1992; Guerra, 2004; Bright y Torres, 2006; Wood, 2007; Salgado, 2008). Hábitos alimenticios: xilomicetofagia y polifagia (Wood, 1982).

90. cf. Amasa sp. 
Nota: tres especímenes capturados en Isla Barro Colorado por medio de trampa Pensilvania de luz ultravioleta requieren ser confirmados para este género. Los especímenes están en la Colección del Programa Centroamericano de Maestría en Entomología de la Universidad de Panamá. Distribución geográfica conocida: El género Amasa contiene especies que se distribuyen en regiones orientales y australianas (Wood y Bright, 1992; Bright y Skidmore, 1997). Algunas especies presentes en Suramérica se han reportado de Brasil (Flechtmann y Cognato, 2011). Hábitos alimenticios: en las especies de la subtribu Xyleborina: xilomicetofagia (Wood, 2007).

\section{Subtribu Cryphalina}

91. Cryptocarenus diadematus Eggers, 1937

Registro para Isla Barro Colorado: nuevo registro. Distribución geográfica conocida: América: Bolivia, Brasil Colombia, Costa Rica, Jamaica, México, Panamá, Venezuela (Wood, 1982; Wood y Bright, 1992; Wood, 2007; Salgado, 2008). Hábitos alimenticios: mielofagia y polifagia (Wood, 1982; Atkinson y Equihua, 1986b).

92. Hypothenemus ascitus Wood, 1971

Registro para Isla Barro Colorado: Wood (1982). Distribución geográfica conocida: América: Costa Rica, Panamá (Wood, 1982). Hábitos alimenticios: fleofagia, mielofagia y polifagia (Wood, 1982).

93. Hypothenemus obscurus (F., 1801)

Registro para Isla Barro Colorado: nuevo registro. Distribución geográfica conocida: América: Brasil, Colombia, Costa Rica, Cuba, Ecuador, Estados Unidos, Guadalupe, Guyana, Jamaica, Panamá, Puerto Rico, República Dominicana, Surinam, Trinidad y Tobago, Venezuela; Asia: Indonesia. Europa: Reino Unido (Hopkins, 1915; Wood, 1982; Wood y Bright, 1992; Guerra, 2004; Bright y Torres, 2006). Hábitos alimenticios: mielofagia y polifagia (Wood, 1982).

94. Hypothenemus plumeriae (Nordlinger) 1856

Registro para Isla Barro Colorado: Wood (1982). Distribución geográfica conocida: África: Liberia, Zaire; América: Brasil, Colombia, Costa Rica, Ecuador, Guatemala, Guadalupe, Honduras, México, Panamá, República Dominicana, Trinidad y Tobago, Venezuela; Europa: Reino Unido (Hopkins, 1915; Wood, 1982; Wood y Bright, 1992; Bright y Skidmore, 2002; Wood, 2007). Hábitos alimenticios: mielofagia y polifagia (Wood, 1982).

95. Hypothenemus suspectus Wood, 1974

Registro para Isla Barro Colorado: Wood (1982). Distribución geográfica conocida: América: Costa Rica, México, Panamá, Venezuela (Wood, 1982; Wood y Bright, 1992; Wood, 2007). Hábitos alimenticios: fleofagia y mielofagia, polifagia (Wood, 1982).

96. Hypothenemus trivialis Wood, 1974

Registro para Isla Barro Colorado: Wood (1974), Wood (1982). Distribución geográfica conocida: América: Colombia, Costa Rica, México, Panamá, Venezuela 
(Wood, 1982; Wood y Bright, 1992). Hábitos alimenticios: fleofagia y mielofagia, polifagia (Wood, 1982).

\section{Subtribu Pityophthorina}

97. Araptus costaricencis (Schedl, 1938)

Registro para Isla Barro Colorado: Wood (1982). Distribución geográfica conocida: América: Brasil, Costa Rica, Panamá (Wood, 1982; Wood, 2007). Hábitos alimenticios: fleofagia y polífagia (Wood, 1982).

98. Araptus eruditus (Schedl, 1938)

Registro para Isla Barro Colorado: Wood (1982). Distribución geográfica conocida: América: Costa Rica, Guatemala, Honduras, México, Panamá (Wood, 1982). Hábitos alimenticios: fleofagia y monofagia (Wood, 1982).

99. Araptus guyanensis Wood, 2007

Registro para Isla Barro Colorado: Wood (2007), Bright (2014). Distribución geográfica conocida: América: Brasil, Guyana Francesa, Panamá (Wood, 2007). Hábitos alimenticios: fleofagia, un solo hospedero reportado (Wood, 1982).

100. Araptus hymenaeae (Eggers, 1933)

Registro para Isla Barro Colorado: Wood (1982). Distribución geográfica conocida: América: Brasil, Colombia, Guadalupe, Guyana, Guyana Francesa, Honduras, Jamaica, Panamá, Puerto Rico, República Dominicana, San Vicente y Las Granadinas, Santa Lucia, Surinam, Trinidad y Tobago, Venezuela; Europa: Reino Unido (Blackman, 1942; Bright, 1981b; Bright, 1982; Wood, 1982; Wood y Bright, 1992; Bright y Skidmore, 2002; Bright y Torres, 2006). Hábitos alimenticios: espermatofagia y oligofagia (Wood, 1982).

101. Araptus laevigatus (Eggers, 1933)

Registro para Isla Barro Colorado: Wood (1982). Distribución geográfica conocida: América: Brasil, Colombia, Costa Rica, Guyana Francesa, Panamá, Surinam (Wood, 1982; Wood y Bright, 1992; Wood, 2007). Hábitos alimenticios: espermatofagia y oligofagia (Wood, 1982).

102. Araptus teres (Blackman, 1942)

Registro para Isla Barro Colorado: nuevo registro. Distribución geográfica conocida: América: Panamá (Wood, 1982). Hábitos alimenticios: fleofagia, hospederos no conocidos (Wood, 1982).

103. Araptus politus (Blandford, 1904)

Registro para Isla Barro Colorado: nuevo registro. Distribución geográfica conocida: América: Costa Rica, Cuba, Haití, Jamaica, México, Panamá (nuevo registro) (Wood, 1982; Atkinson, y Equihua, 1986). Hábitos alimenticios: espermatofagia y monofagia (Wood, 1982).

104. Araptus cf. A. conditus 
Nota: diez especímenes capturados en Isla Barro Colorado con trampa Pensilvania de luz ultravioleta requieren ser confirmados para esta especie. Los especímenes están en la Colección del Programa Centroamericano de Maestría en Entomología de la Universidad de Panamá. Distribución geográfica conocida: Araptus conditus Wood, 1974: América: Costa Rica (Wood, 1974; Wood, 1982). Hábitos alimenticios: en Araptus conditus: fleofagia (Wood, 1982).

\section{Araptus cf. A. laudatus}

Nota: 33 especímenes capturados en Isla Barro Colorado con trampa Pensilvania de luz ultravioleta requieren ser confirmados para esta especie. Los especímenes están en la Colección del Programa Centroamericano de Maestría en Entomología de la Universidad de Panamá. Distribución geográfica conocida: Araptus laudatus Wood, 1974: América: Costa Rica, México (Wood, 1974; Wood, 1982; Atkinson y Equihua, 1986b; Bright y Skidmore, 2002). Hábitos alimenticios: en Araptus laudatus: fleofagia y monofagia (Wood, 1982).

106. Araptus sp.

Nota: nueve especímenes capturados en Isla Barro Colorado con trampa Pensilvania de luz ultravioleta. Los especímenes están en la Colección del Programa Centroamericano de Maestría en Entomología de la Universidad de Panamá. Distribución geográfica conocida: del género Araptus, 54 especies ocurren de México a Centroamérica (Wood, 1982) y 53 en Suramérica (Wood 2007). Hábitos alimenticios: en las especies del género Araptus: fleofagia y espermatofagia (Wood, 1982).

107. Gnatholeptus panamensis Blackman, 1943

Registro para Isla Barro Colorado: Bright (1981b), Wood (1982), Wood y Bright (1992). Distribución geográfica conocida: América: Costa Rica, Panamá, Surinam (Wood, 1982). Hábitos alimenticios: fleofagia, hospederos no conocidos (Wood, 1982).

108. Gnatholeptus shannoni (Blackman, 1942)

Registro para Isla Barro Colorado: Bright (1981b), Wood (1982), Wood y Bright (1992). Distribución geográfica conocida: Colombia, Panamá (Bright, 1981b; Wood, 1982). Hábitos alimenticios: fleofagia y monofagia (Wood, 1982).

109. Pityophthorus dimidiatus Blackman, 1942

Registro para Isla Barro Colorado: nuevo registro. Distribución geográfica conocida: América: México, Panamá (nuevo registro) (Bright, 1981b). Hábitos alimenticios: fleofagia, hospederos no conocidos (Wood, 2007).

110. Pityophthorus vesculus Wood, 1978

Registro para Isla Barro Colorado: nuevo registro. Distribución geográfica conocida: América: Panamá (Wood, 1978; Bright, 1981a; Wood, 1982). Hábitos alimenticios: fleofagia, hospederos no conocidos (Wood, 2007).

\section{Pityophthorus cf. P. galeritus}

Nota: un espécimen capturados en Isla Barro Colorado con trampa Pensilvania de luz ultravioleta requiere ser confirmados para esta especie. El espécimen está en la Colección 
del Programa Centroamericano de Maestría en Entomología de la Universidad de Panamá. Distribución geográfica conocida: Pityophthorus galeritus Wood, 1976: América: Costa Rica (Bright, 1981b; Wood, 1982; Wood y Bright, 1992). Hábitos alimenticios: en las especies del género Pityophthorus: fleofagia (Wood, 2007).

\section{Pityophthorus cf. P. nemoralis}

Nota: un espécimen capturados en Isla Barro Colorado con trampa Pensilvania de luz ultravioleta requiere ser confirmados para esta especie. El espécimen está en la Colección del Programa Centroamericano de Maestría en Entomología de la Universidad de Panamá. Distribución geográfica conocida: Pityophthorus nemoralis Wood, 1976: América: Costa Rica, Honduras (Bright, 1981b; Wood, 1982; Wood y Bright, 1992). Hábitos alimenticios: en las especies del género Pityophthorus: fleofagia (Wood, 2007).

113. Pityophthorus cf. P. perexiguus

Nota: siete especímenes capturados en Isla Barro Colorado con trampa Pensilvania de luz ultravioleta requiere ser confirmados para esta especie. Los especímenes están en la Colección del Programa Centroamericano de Maestría en Entomología de la Universidad de Panamá. Distribución geográfica conocida: Pityophthorus perexiguus Wood, 1976: América: Costa Rica, Panamá (Bright, 1981b; Wood, 1982). Hábitos alimenticios: en las especies del género Pityophthorus: fleofagia (Wood, 2007).

\section{Pityophthorus cf. P. strictus}

Nota: 16 especímenes capturados en Isla Barro Colorado con trampa Pensilvania de luz ultravioleta requieren ser confirmados para esta especie. Los especímenes están en la Colección del Programa Centroamericano de Maestría en Entomología de la Universidad de Panamá. Distribución geográfica conocida: Pityophthorus strictus Wood, 1976: América: Costa Rica (Bright, 1981a; Wood, 1982; Wood y Bright, 1992). Hábitos alimenticios: en las especies del género Pityophthorus: fleofagia (Wood, 2007).

\section{Pityophthorus (Pityophthorus) sp. Grupo Alni}

Nota: un espécimen capturado en Isla Barro Colorado con trampa Pensilvania de luz ultravioleta. El espécimen está en la Colección del Programa Centroamericano de Maestría en Entomología de la Universidad de Panamá. Distribución geográfica conocida: las especies del grupo Alni ocurren en la Región Neotropical, nueve especies reportadas para este grupo en Norte y Centroamérica (Bright, 1981a). Hábitos alimenticios: en las especies del género Pityophthorus: fleofagia (Wood, 2007).

\section{Subtribu Corthylina}

116. Amphicranus argutus Wood, 1976

Registro para Isla Barro Colorado: nuevo registro. Distribución geográfica conocida: América: Costa Rica, Panamá (nuevo registro) (Wood, 1982). Hábitos alimenticios: xilomicetofagia y polifagia (Wood, 2007).

\section{Amphicranus macellus Wood, 1974}


Registro para Isla Barro Colorado: nuevo registro. Distribución geográfica conocida: América: Costa Rica, Panamá (nuevo registro) (Wood, 1982). Hábitos alimenticios: xilomicetofagia y polifagia (Wood, 2007).

118. Amphicranus micans Wood, 1976

Registro para Isla Barro Colorado: nuevo registro. Distribución geográfica conocida: América: Costa Rica, México, Panamá (Wood, 1982; Atkinson, y Equihua, 1986). Hábitos alimenticios: xilomicetofagia y polifagia (Wood, 2007).

119. Amphicranus spectabilis (Wood, 1967)

Registro para Isla Barro Colorado: nuevo registro. Distribución geográfica conocida: América: Costa Rica, Panamá (nuevo registro) (Wood, 1982). Hábitos alimenticios: xilomicetofagia y polifagia (Wood, 2007).

120. Corthylocurus debilis Wood, 1974

Registro para Isla Barro Colorado: nuevo registro. Distribución geográfica conocida: América: Costa Rica, México, Panamá (Wood, 1982; Estrada y Atkinson, 1989). Hábitos alimenticios: xilomicetofagia y polifagia (Wood, 2007).

121. Corthyloxiphus cf. C. furvus

Nota: tres especímenes capturados en Isla Barro Colorado con trampa Pensilvania de luz ultravioleta requieren ser confirmados para esta especie. Los especímenes están en la Colección del Programa Centroamericano de Maestría en Entomología de la Universidad de Panamá. Distribución geográfica conocida: Corthyloxiphus furvus (Wood, 1974): América: Costa Rica (Wood, 1982). Hábitos alimenticios: en las especies del género Corthyloxiphus: xilomicetofagia (Wood, 2007).

122. Corthylus atomus Wood, 2007

Registro para Isla Barro Colorado: Wood (2007), Bright (2014). Distribución geográfica conocida: América: Panamá (Wood, 2007; Bright, 2014). Hábitos alimenticios: xilomicetofagia y polifagia (Wood, 2007).

123. Corthylus procerus Bright, 1972

Registro para Isla Barro Colorado: nuevo registro. Distribución geográfica conocida: América: Costa Rica, México, Panamá (Bright, 1972; Wood, 1982). Hábitos alimenticios: xilomicetofagia y polifagia (Wood, 2007).

124. Corthylus pygmaeus Wood, 1974

Registro para Isla Barro Colorado: Atkinson y Equihua (1988). Distribución geográfica conocida: América: Costa Rica, Panamá (Wood, 1982; Atkinson y Equihua, 1988). Hábitos alimenticios: xilomicetofagia y polifagia (Wood, 2007).

125. Corthylus sobrinus Wood, 1974

Registro para Isla Barro Colorado: nuevo registro. Distribución geográfica conocida: América: Costa Rica, Panamá (nuevo registro) (Wood, 1982). Hábitos alimenticios: xilomicetofagia y polifagia (Wood, 2007). 
126. Corthylus cf. C. nanus

Nota: un espécimen capturados en Isla Barro Colorado con trampa Pensilvania de luz ultravioleta requiere ser confirmados para esta especie. El espécimen está en la Colección del Programa Centroamericano de Maestría en Entomología de la Universidad de Panamá. Distribución geográfica conocida: Corthylus nanus Wood, 1979: América: Costa Rica (Wood, 1982). Hábitos alimenticios: en las especies de Corthylus: xilomicetofagia y polifagia (Wood, 2007).

\section{Corthylus cf. C. nevermanni}

Nota: 15 especímenes capturados en Isla Barro Colorado con trampa Pensilvania de luz ultravioleta requieren ser confirmados para esta especie. Los especímenes están en la Colección del Programa Centroamericano de Maestría en Entomología de la Universidad de Panamá. Distribución geográfica conocida: Corthylus nevermanni (Schedl, 1939): América: Costa Rica (Wood, 2007). Hábitos alimenticios: en las especies de Corthylus: xilomicetofagia y polifagia (Wood, 2007).

\section{Corthylus cf. C. panamensis}

Nota: dos especímenes capturados en Isla Barro Colorado con trampa Pensilvania de luz ultravioleta requieren ser confirmados para esta especie. Los especímenes están en la Colección del Programa Centroamericano de Maestría en Entomología de la Universidad de Panamá. Distribución geográfica conocida: Corthylus panamensis Blandford, 1904: América: Guatemala, Costa Rica, Panamá (Wood, 1982). Hábitos alimenticios: en las especies de Corthylus: xilomicetofagia y polifagia (Wood, 2007).

129. Corthylus cf. C. papulans

Nota: dos especímenes capturados en Isla Barro Colorado con trampa Pensilvania de luz ultravioleta requieren ser confirmados para esta especie. Los especímenes están en la Colección del Programa Centroamericano de Maestría en Entomología de la Universidad de Panamá. Distribución geográfica conocida: Corthylus papulans Eichhoff, 1869: América: Honduras, Costa Rica (Wood, 1982). Hábitos alimenticios: en las especies de Corthylus: xilomicetofagia y polifagia (Wood, 2007).

130. Corthylus cf. C. pumilus

Nota: un espécimen capturados en Isla Barro Colorado con trampa Pensilvania de luz ultravioleta requiere ser confirmados para esta especie. El espécimen está en la Colección del Programa Centroamericano de Maestría en Entomología de la Universidad de Panamá. Distribución geográfica conocida: Corthylus pumilus Wood, 1974: América: México, Panamá (Wood, 1982; Bright y Skidmore, 1997). Hábitos alimenticios: en las especies de Corthylus: xilomicetofagia y polifagia (Wood, 2007).

131. Corthylus sp.

Nota: dos especímenes capturados en Isla Barro Colorado con trampa Pensilvania de luz ultravioleta. Los especímenes están en la Colección del Programa Centroamericano de Maestría en Entomología de la Universidad de Panamá. Distribución geográfica conocida: en Centroamérica el género está representado por 40 especies (Wood y Bright, 1992), 20 reportadas para Panamá (Wood, 1982; Atkinson y Equihua, 1988; Wood y 
Bright, 1992; Wood, 2007). Hábitos alimenticios: en las especies de Corthylus: xilomicetofagia y polifagia (Wood, 2007).

132. Metacorthylus mutilus (Wood, 1974)

Registro para Isla Barro Colorado: nuevo registro. Distribución geográfica conocida: América: Panamá (Wood, 1974; Wood, 1982). Hábitos alimenticios: xilomicetofagia y polifagia (Wood, 2007).

133. Monarthrum dolosum Wood, 2007

Registro para Isla Barro Colorado: nuevo registro. Distribución geográfica conocida: América: Colombia, Costa Rica, Panamá, Venezuela (Wood, 1982; Wood, 2007). Hábitos alimenticios: xilomicetofagia y polifagia (Wood, 2007).

134. Monarthrum exornatum (Schedl, 1939)

Registro para Isla Barro Colorado: nuevo registro. Distribución geográfica conocida: América: Colombia, Costa Rica, México, Panamá (nuevo registro), Venezuela; Europa: Reino Unido (Wood, 1982; Wood y Bright, 1992; Wood, 2007). Hábitos alimenticios: xilomicetofagia y polifagia (Wood, 2007).

135. Monarthrum posticum Wood, 1974

Registro para Isla Barro Colorado: nuevo registro. Distribución geográfica conocida: América: Costa Rica, Panamá (nuevo registro) (Wood, 1982). Hábitos alimenticios: xilomicetofagia y polifagia (Wood, 2007).

136. Monarthrum sp.

Nota: dos especímenes capturados en Isla Barro Colorado por medio de trampa Pensilvania de luz ultravioleta. Distribución geográfica conocida: especies del género Monarthrum se encuentran desde Estados Unidos hasta Argentina, 118 especies ocurren en Norte y Centroamérica; 17 especies reportadas de Panamá (Wood, 1982; Wood, 2007). Hábitos alimenticios: todas las especies del género son xilomicetófagas (Wood, 2007).

137. Tricolus badius Wood, 1974

Registro para Isla Barro Colorado: nuevo registro. Distribución geográfica conocida: América: Costa Rica, México, Panamá (Wood, 1974; Wood, 1982; Bright y Skidmore, 1997). Hábitos alimenticios: xilomicetofagia y polifagia (Wood, 2007).

138. Tricolus plaumanni Schedl, 1954

Registro para Isla Barro Colorado: nuevo registro. Distribución geográfica conocida: América: Brasil, Costa Rica, Panamá (Wood, 1982). Hábitos alimenticios: xilomicetofagia, hospederos no conocidos (Wood, 2007).

Subfamilia Platypodinae

\author{
Tribu Mecopelmini \\ Subtribu Mecopelmina
}

139. Mecopelmus zeteki Blackman, 1944 
Registro para Isla Barro Colorado: Wood y Bright (1992). Distribución geográfica conocida: América: Costa Rica, Panamá, Perú (Wood, 1966; Equihua y Atkinson, 1987; Wood y Bright, 1992; Bright y Skidmore, 2002). Hábitos alimenticios: xilofagia, solo una especie de hospedero conocido (Wood, 1993).

\section{Tribu Tesserocerini Subtribu Tesserocerina}

140. Cenocephalus sp.

Nota: un espécimen capturado en Isla Barro Colorado con trampa Pensilvania de luz ultravioleta. Los especímenes están en la Colección del Programa Centroamericano de Maestría en Entomología de la Universidad de Panamá. Distribución geográfica conocida: dos especies de Conocephalus se han reportado para Centroamérica: $C$. epistomalis y C. lalolaensis, ninguno de ellas reportadas para Panamá (Wood, 1966; Wood y Bright, 1992). Hábitos alimenticios: xilomicetofagia (Wood, 1982; Wood, 1993).

141. Tesserocerus dewalquei Chapuis, 1865

Registro para Isla Barro Colorado: Reichardt (1965), Equihua y Atkinson (1987) Rodríguez y Barrios (2017). Distribución geográfica conocida: América: Argentina, Bolivia, Brasil, Colombia, Costa Rica, El Salvador, Guatemala, Guyana, Guyana Francesa, México, Nicaragua, Panamá, Paraguay, Perú, Surinam, Venezuela (Reichardt, 1965; Equihua y Atkinson, 1987; Wood y Bright, 1992; Bright y Skidmore, 2002; Rodríguez y Barrios, 2017). Hábitos alimenticios: xilomicetofagia y polifagia (Wood, 1982; Wood, 1993).

\section{Tesserocerus ericius Blandford, 1895}

Registro para Isla Barro Colorado: Reichardt (1965), Equihua y Atkinson (1987), Rodríguez y Barrios (2017). Distribución geográfica conocida: América: Belice, Brasil, Costa Rica, Guatemala, México, Panamá, Perú (Reichardt, 1965; Equihua y Atkinson, 1987; Wood y Bright, 1992, Kirkendall, 2017; Rodríguez y Barrios, 2017). Hábitos alimenticios: xilomicetofagia y polifagia (Wood, 1982; Wood, 1993).

143. Tesserocerus retusus Guerin-Meneville, 1838

Registro para Isla Barro Colorado: nuevo registro. Distribución geográfica conocida: América: México, Colombia, Panamá (nuevo registro) (Wood y Bright, 1992). Hábitos alimenticios: xilomicetofagia, hospederos no conocidos (Wood, 1982; Wood, 1993).

\section{Tribu Platypodini Subtribu Platypodina}

144. Costaroplatus pulchellus (Chapuis, 1865)

Registro para Isla Barro Colorado: Rodríguez y Barrios (2017). Distribución geográfica conocida: América: El Salvador, Guatemala, Guyana, México, Nicaragua, Panamá, Surinam, Venezuela; Europa: Reino Unido (Equihua, 1985; Wood y Bright, 1992; Rodríguez y Barrios, 2017). Hábitos alimenticios: xilomicetofagia y polifagia (Wood, 1982; Wood, 1993). 
145. Euplatypus parallelus (F., 1801)

Registro para Isla Barro Colorado: Rodríguez y Barrios (2017). Distribución geográfica conocida: África: Angola, Camerún, Costa de Marfil, Gabón, Ghana, Guinea, Guinea Ecuatorial, Kenia, Liberia, Madagascar, Nigeria, Santo Tomé y Príncipe, Senegal, Sierra Leona, Tanzania, Togo, Uganda, Zaire. América: Argentina, Belice, Brasil, Chile, Colombia, Cuba, Ecuador, El Salvador, Estados Unidos, Guadalupe, Guatemala, Guyana, Haití, Islas Vírgenes, México, Nicaragua, Panamá, Perú, República Dominicana, Uruguay, Venezuela; Europa: Inglaterra; Oceanía: Australia (Equihua, 1985; Wood y Bright, 1992; Kirkendall, 2017; Rodríguez y Barrios, 2017). Hábitos alimenticios: xilomicetofagia y polifagia (Wood, 1982; Wood, 1993).

146. Euplatypus cf. E. segnis

Nota: 266 especímenes capturados en Isla Barro Colorado con trampa Pensilvania de luz ultravioleta. Los especímenes están en la Colección del Programa Centroamericano de Maestría en Entomología de la Universidad de Panamá. Distribución geográfica conocida: Euplatypus segnis (Chapuis, 1865): América: Colombia, Costa Rica, Guadalupe, México, Perú y Venezuela (Estrada y Atkinson, 1989; Wood y Bright, 1992). Hábitos alimenticios: en las especies de Euplatypus: xilomicetofagia y polifagia (Wood, 1982; Wood, 1993).

\section{Euplatypus cf. E. compositus}

Nota: un espécimen capturados en Isla Barro Colorado con trampa Pensilvania de luz ultravioleta requiere ser confirmado para esta especie. El espécimen está en la Colección del Programa Centroamericano de Maestría en Entomología de la Universidad de Panamá.

Distribución geográfica conocida: Euplatypus compositus (Say, 1823): África: Madagascar. América: Argentina, Brasil, Costa Rica, Estados Unidos, Guyana Francesa, México, Venezuela (Staines, 1982, Estrada y Atkinson, 1989; Wood y Bright, 1992). Hábitos alimenticios: en las especies de Euplatypus: xilomicetofagia y polifagia (Wood, 1982; Wood, 1993).

\section{Euplatypus $\mathrm{cf}$. E. cribicollis}

Nota: seis especímenes capturados en Isla Barro Colorado con trampa Pensilvania de luz ultravioleta requieren ser confirmados para esta especie. Los especímenes están en la Colección del Programa Centroamericano de Maestría en Entomología de la Universidad de Panamá. Distribución geográfica conocida: Euplatypus cribricollis (Blandford, 1896): América: Panamá (Wood y Bright, 1992). Hábitos alimenticios: en las especies de Euplatypus: xilomicetofagia y polifagia (Wood, 1982; Wood, 1993).

149. Megaplatypus cf. M. deyrollei

Nota: cinco especímenes capturados en Isla Barro Colorado con trampa Malaise requieren ser confirmados para esta especie. Los especímenes están en la Colección del Programa Centroamericano de Maestría en Entomología de la Universidad de Panamá. Distribución geográfica conocida: Megaplatypus deyrollei (Chapuis, 1865): América: Guatemala, México y Panamá (Blandford, 1905; Wood y Bright, 1992). Hábitos alimenticios: en las especies de Megaplatypus: xilomicetofagia y polifagia (Wood, 1982; Wood y Bright, 1992; Wood, 1993). 
150. Megaplatypus cf. M. exaratus

Nota: cinco especímenes capturados en Isla Barro Colorado con trampa Malaise requieren ser confirmados para esta especie. Los especímenes están en la Colección del Programa Centroamericano de Maestría en Entomología de la Universidad de Panamá. Distribución geográfica conocida: Megaplatypus exaratus (Blandford, 1895): América: Guatemala y México (Blandford, 1905; Atkinson, et al. 1986). Hábitos alimenticios: en las especies de Megaplatypus: xilomicetofagia y polifagia (Wood, 1982; Wood y Bright, 1992; Wood, 1993).

151. Megaplatypus cf. M. limbatus

Nota: 16 especímenes capturados en Isla Barro Colorado con trampas Malaise y Pensilvania con luz ultravioleta requieren ser confirmados para esta especie. Los especímenes están en la Colección del Programa Centroamericano de Maestría en Entomología de la Universidad de Panamá. Distribución geográfica conocida: Megaplatypus limbatus (Chapuis, 1865): América: Costa Rica y Brasil, Guatemala, México (Blandford, 1905; Wood y Bright, 1992). Hábitos alimenticios: en las especies de Megaplatypus: xilomicetofagia y polifagia (Wood, 1982; Wood y Bright, 1992; Wood, 1993).

\section{Megaplatypus cf. M. mutatus}

Nota: 14 especímenes capturados en Isla Barro Colorado con trampas Malaise y Pensilvania con luz ultravioleta requieren ser confirmados para esta especie. Los especímenes están en la Colección del Programa Centroamericano de Maestría en Entomología de la Universidad de Panamá. Distribución geográfica conocida: Megaplatypus mutatus (Chapius, 1865): América: Argentina, Brasil, Bolivia, Guayana Francesa, Paraguay, Perú, Uruguay, Venezuela (Giménez, 2009). Hábitos alimenticios: en las especies de Megaplatypus: xilomicetofagia y polifagia (Wood, 1982; Wood y Bright, 1992; Wood, 1993).

\section{Megaplatypus sp.}

Nota: nueve especímenes capturados en Isla Barro Colorado con trampa Malaise y Pensilvania de luz ultravioleta fueron organizados en tres morfoespecies diferentes a las especies de Megaplatypus encontradas en el material identificado. Los especímenes están en la Colección del Programa Centroamericano de Maestría en Entomología de la Universidad de Panamá. Distribución geográfica conocida: seis especies reportadas para Panamá: $M$. chiriquensis, $M$. deyrollei, $M$. dolabratus, $M$. imporcatus, $M$. porrectus y $M$. ramali (Wood, 1966; Blandford, 1905; Wood y Bright, 1992). Hábitos alimenticios: en las especies de Megaplatypus: xilomicetofagia y polifagia (Wood, 1982; Wood y Bright, 1992; Wood, 1993).

\section{Platyphysus sp.}

Nota: un espécimen capturado en Isla Barro Colorado con trampa Pensilvania de luz ultravioleta. Los especímenes están en la Colección del Programa Centroamericano de Maestría en Entomología de la Universidad de Panamá. Distribución geográfica conocida: para las especies del género Platyphysus: América: Costa Rica a Brasil (Wood, 1993). Hábitos alimenticios: xilomicetofagia (Wood, 1982; Wood, 1993). 
155. Teloplatypus excisus (Chapuis, 1865)

Registro para Isla Barro Colorado: nuevo registro. Distribución geográfica conocida: América: Argentina, México, Panamá (nuevo registro), Perú, República Dominicana (Equihua, 1985; Wood y Bright, 1992; Bright y Skidmore, 2002, Kirkendall, 2017). Hábitos alimenticios: xilomicetofagia y polifagia (Wood, 1982; Wood, 1993).

156. Teloplatypus ustulatus (Chapuis, 1865)

Registro para Isla Barro Colorado: nuevo registro. Distribución geográfica conocida: América: Brasil, Colombia, Guyana Francesa, Jamaica, México, Panamá (nuevo registro), República Dominica (Wood y Bright, 1992; Bright y Skidmore, 1997). Hábitos alimenticios: xilomicetofagia y polifagia (Wood, 1982; Wood, 1993).

\section{Teloplatypus sp.}

Nota: diez especímenes capturados en Isla Barro Colorado con trampa Malaise y Pensilvania de luz ultravioleta fueron organizados en dos morfoespecies diferentes a las especies de Teloplatypus encontradas en el material identificado. Los especímenes están en la Colección del Programa Centroamericano de Maestría en Entomología de la Universidad de Panamá. Distribución geográfica conocida: las especies $T$. marcidus y T. pallidipennis están reportadas para Panamá (Blandford, 1905; Wood y Bright, 1992). Hábitos alimenticios: en las especies de Teloplatypus: xilomicetofagia y polifagia (Wood, 1982; Wood y Bright, 1992; Wood, 1993).

158. cf. Neotrachyostus sp.

Nota: 175 especímenes capturados en Isla Barro Colorado por medio de trampa Pensilvania de luz ultravioleta requieren ser confirmados para este género. Los especímenes están en la Colección del Programa Centroamericano de Maestría en Entomología de la Universidad de Panamá. Distribución geográfica conocida: 14 especies conocidas con distribución del Sureste de México a Brasil, no se han reportado representantes del género para Panamá (Schedl, 1972; Wood y Bright, 1992; Wood, 1993; Attkinson, 2018). Hábitos alimenticios: en las especies de Neotrachyostus: xilomicetofagia (Wood, 1982; Wood y Bright, 1992; Wood, 1993).

\section{CONCLUSIONES}

Un total de 48 especies de Scolytinae y 5 especies de Platypodinae se han registrado de la Isla Barro Colorado en diferentes publicaciones científicas.

La presente investigación aporta 64 especies de Scolytinae y 3 especies de Platypodinae como nuevos registros para la isla. De estos nuevos registros, 29 especies de Scolytinae y tres de Platypodinae son nuevos registros para Panamá.

La cantidad de especies de Scolytinae encontradas en BCI es de 113, mientras que los Platypodinae alcanza las 8. Sin embargo, estas cifras pueden aproximarse a 138 especies para Scolytinae y 20 para Platypodinae. 


\section{SUMMARY}

SCOLYTINAE AND PLATYPODINAE (COLEOPTERA: CURCULIONIDAE) OF THE BARRO COLORADO ISLAND, PANAMA.

Isla Barro Colorado (BCI) is a natural reserve located in the Gatun Lake of the Panama Canal, it has been an important tropical area for studies in biology and tropical ecology. However, certain biological groups do not have fauna inventories, one of them is the ambrosial beetles (Coleoptera: Curculionidae). For this reason, he carried out an enriched inventory of the Scolytinae and Platypodinae reported for BCI. To develop the inventory, the following was carried out: 1) review of scientific literature with citations of species registration in the island, 2) review of specimens of the entomological collections of the Universidad de Panama and 3) taxonomic identification of specimens in samples captured by the Smithsonian Research Tropical Institute. A total of 48 species of Scolytinae and 3 species of Platypodinae had been recorded from the island through scientific publications. The examination, taxonomic identification and the database provided 64 species of Scolytinae and three species of Platypodinae as new records for the island. The number of Scolytinae species known for the island reaches 113 species, while the Platypodinae reach 8 species; however, with the taxonomic confirmation of some species, these figures can approach 138 species for Scolytinae and 20 for Platypodinae.

\section{KEYWORDS}

Ambrosia beetles, Species inventory, New insect records from Panama, Xylomycetophagy, Fungus-farming insects.

\section{REFERENCIAS BIBLIOGRÁFICAS}

ALFARO, R.I., HUMBLE, L.M., GONZALEZ, P. VILLAVERDE, R. y ALLEGRO, G. 2007. The threat of the ambrosia beetle Megaplatypus mutatus (Chapuis) (=Platypus mutatus Chapuis) to world poplar resources. Forestry, 80, 471-479.

ALONSO-ZARAZAGA, M. y LYAL, C.H.C. 2009. A catalogue of family and genus group names in Scolytinae and Platypodinae with nomenclatural remarks (Coleoptera: Curculionidae). Zootaxa, 2258, 1-34.

ATKINSON, T.H. 1989. New species and notes on Mexican Hylesininae (Coleoptera: Scolytidae). Insecta Mundi, 3 (1), 57-64.

ATKINSON, T.H. 2017. Familia Curculionidae: subfamilia Platypodinae. En: Fundamentos de Entomología Forestal. Ed: Cibrián T.D. p. 314-315. México: Universidad Autónoma de Chapingo. 
ATKINSON, T.H. 2018. A new species, new synonymy and new records of Mexican and Central American Xyleborini (Coleoptera: Curculionidae: Scolytinae). Zootaxa, 4442 (2): 345-350.

ATKINSON, T.H. 2018. Bark and Ambrosia Beetles (en línea). Disponible en http://www.barkbeetles.info

ATKINSON, T.H. y EQUIHUA M, A. 1985. Notes on biology and distribution of Mexican and Central American Scolytidae (Coleoptera). II. Scolytinae: Cryphalini and Corthylini. The Coleopterists Bulletin, 39, 355-363.

ATKINSON, T.H. y EQUIHUA M, A. 1986a. Biology of the Scolytidae and Platypodidae (Coleoptera) in a tropical deciduous forest at Chamela, Jalisco, Mexico. Florida Entomologist, 69 (2), 303-310.

ATKINSON, T.H. y EQUIHUA M, A. 1986b. Biology of bark and ambrosia beetles (Coleoptera: Scolytidae and Platypodidae) of a tropical rain forest in southeastern Mexico with an annotated checklist of species. Annals of the Entomological Society of America, 79 (3), 414-423.

ATKINSON, T.H. y EQUIHUA M., A. 1988. Notas sobre la biología de Scolytidae y Platypodidae de México y Centroamérica. Folia Entomológica Mexicana, 76, 83105.

ATKINSON, T.H. Y RABAGLIA, R.J. 2010. Listado de especies de Scolytinae (Curculionidae) de Centroamérica. Curso de Identificación de Coleópteros Descortezadores y Ambrosiales. Universidad Nacional Agraria. 22 pp. (En manuscrito)

ATKINSON, T.H. y RILEY, E.G. 2013. Atlas and Checklist of the bark and ambrosia beetles of Texas and Oklahoma (Curculionidae: Scolytinae and Platypodinae). Insecta Mundi, 292, 1-46.

ATKINSON, T.H., CARRILLO, D., DUNCAN, R.E. y PEÑA, J.E. 2013. Occurrence of Xyleborus bispinatus (Coleoptera: Curculionidae: Scolytinae) Eichhoff in southern Florida. Zootaxa, 3669 (1), 96-100.

ATKINSON, T.H., MARTINEZ F, E., SAUCEDO C, E. y BURGOS S, A. 1986. Scolytidae y Platypodidae (Coleoptera) asociados a selva baja caducifolia y comunidades derivadas en el estado de Morelos. Folia Entomológica Mexicana, 69, 41-82.

ATKINSON, T.H., RABAGLIA, R.J. y COGNATO, A.I. 2009. Two newly detected bark and ambrosia beetles from southern Florida (Coleoptera: Curculionidae: Scolytinae). Zootaxa, 2311, 66-68.

BEAVER, R.A., GHAHARI, H. y SANGUANSUB, S. 2016. An annotated checklist of Platypodinae and Scolytinae (Coleoptera: Curculionidae) from Iran. Zootaxa, 4098 (3), 401-441. 
BLACKMAN, M.W. 1942. Revision of the bark beetles belonging to the genus Pseudohylesinus Swaine, Publications 461. Washington DC: Department Agriculture, $32 \mathrm{pp}$.

BLANDFORD, W.F.H. 1905. Fam. Scolytidae. Biol.Centrali-Americana, 4 (6), 81-298.

BRIGHT, D.E. 1972. New species of Scolytidae (Coleoptera) from Mexico, with additional notes. I. Tribes Xyleborini and Corthylini. The Canadian Entomologist, 104, 1369-1385.

BRIGHT, D.E. 1981a. Taxonomic monograph of the genus Pityophthorus Eichhoff in North and Central America (Coleoptera: Scolytidae). Memoirs of the Entomological Society of Canada, 113 (118), 1-378.

BRIGHT, D.E. 1981b. Studies on West Indian Scolytidae (Coleoptera) 1. New species, new distribution records and taxonomic notes. Studies on Neotropical Fauna and Environment, 16, 151-164.

BRIGHT, D.E. 1982. Scolytidae (Coleoptera) from the Cocos Islands, Costa Rica, with a description of one new species. The Coleopterists Bulletin, 36 (1), 127-130.

BRIGHT, D.E. 2014. A Catalog of Scolytidae and Platypodidae (Coleoptera), Supplement 3 (2000-2010), with notes on subfamily and tribal reclassifications. Insecta Mundi, 0356, 1-336.

BRIGHT, D.E. y SKIDMORE, R.E. 1997. A Catalog of Scolytidae and Platypodidae (Coleoptera), Supplement 1 (1990-1994). Ontario: NRC Research Press. 368 pp.

BRIGHT, D.E. y SKIDMORE, R.E. 2002. A Catalog of Scolytidae and Platypodidae (Coleoptera): Supplement 2 (1995-1999) (Vol. 108109). Ontario: NRC Research Press. 523 pp.

BRIGHT, D.E. y TORRES, J.A. 2006. Studies on West Indian Scolytidae (Coleoptera) 4. A review of the Scolytidae of Puerto Rico, U.S.A. with descriptions of one new genus, fourteen new species and notes on new synonymy (Coleoptera: Scolytidae). Koleopterisches Rundschau, 76, 389-421.

CASAUBON, E., CUETO, G. y SPAGARINO, C. 2006. Diferente comportamiento de Megaplatypus mutatus (=Platypus sulcatus) (CHAPUIS, 1865) en un ensayo comparativo de rendimiento de 30 clones de Populus deltoides Batr. en el bajo Delta Bonaerense del Río Paraná. Revista de Investigaciones Agropecuarias, 35(2), 103-115.

CHAPUIS, F. 1865. Monographie des Platypides. Ed. LIEGE, Bélgica. 369 pp.

CIBRIÁN, T.D., MÉNDEZ M, J.T., CAMPOS B, R., YATES III, H.O. y FLORES, J.E. 1995. Insectos Forestales de México/Forest Insects of Mexico. México: Universidad Autónoma Chapingo. 453 pp. 
CONDIT, R., WATTS, K., BOHLMAN, S.A., PÉREZ, R., HUBBELL, S.P. y FOSTER, R.B. 2000. Quantifying the deciduousness of tropical forest canopies under varying climates. Journal of Vegetation Science, 11 (5), 649-658.

DOUGLAS, H., BOUCHARD, P., ANDERSON, R.S., DE TONNANCOUR, P., VIGNEAULT, R. y WEBSTER, P. 2013. New Curculionoidea (Coleoptera) records for Canada. Zookeys, 309, 14-38.

EQUIHUA M.A. 1985. Nuevos registros de localidades y huéspedes de coleópteros Platypodidae americanos. Folia Entomológica Mexicana, 66, 143-144.

EQUIHUA M.A., y ATKINSON, T.H. 1987. Catálogo de Platypodidae (Coleoptera) de norte y centroamérica. Folia Entomológica Mexicana, 72, 5-31.

ESTRADA V, A. y ATKINSON, T.H. 1989. Scolytidae y Platypodidae de Escárcega, Campeche, México. Biogeografía, biología, importancia económica y una lista de especies. Anales del Instituto de Biología, Serie Zoología, 58 (1), 199-220.

FLECHTMANN, C.A. y COGNATO, A.I. 2011. First Report of Amasa truncata (Erichson) (Coleoptera: Curculionidae: Scolytinae) in Brazil. The Coleopterists Bulletin, 65 (4), 417-421.

FLECHTMANN, C.A.H. y ATKINSON, T.H. 2016. First records of Xylosandrus crassiusculus (Motschulsky) (Coleoptera: Curculionidae: Scolytinae) from South America, with notes on its distribution and spread in the New World. The Coleopterists Bulletin, 70 (1), 79-83.

GIMÉNEZ, R. 2009. Megaplatypus mutatus: bases para su manejo integrado. Bariloche, Argentina. Universidad de Buenos Aires/INTA. 18 pp.

GUERRA, L. 2004. Caracterización de la comunidad de Scolytidae colectadas con diferentes tipos de trampa ubicadas en cinco sitios con predominio de Pino en la República de Panamá. Tesis Magister, Universidad de Panamá, Panamá. 106 pp.

HECKADON-MORENO, S., IBÁÑEZ, R. y CONDIT, R. (Eds) 1999. La Cuenca del Canal: deforestación, urbanización y contaminación. Instituto Smithsoniano de Investigaciones Tropicales, Panamá, Panamá. 120 pp.

HOPKINS, A.D. 1915. Classification of the Cryphalinae with descriptions of new genera and species. Contributions of Bureau Entomology, 99, 1-75.

HULCR, J. y DUNN, R. R. 2011. The sudden emergence of pathogenicity in insectfungus symbioses threatens naive forest ecosystems. Proceedings of the Royal Society of London B: Biological Sciences, 278 (1720), 2866-2873.

HULCR, J. y SMITH, S. 2010. Xyleborini ambrosia beetles: an identification tool to the world genera (en línea). Disponible en http://itp.lucidcentral.org 
JORDAL, B. H. 2015. Molecular phylogeny and biogeography of the weevil subfamily Platypodinae reveals evolutionarily conserved range patterns. Molecular phylogenetics and evolution, 92, 294-307.

JORDAL, B.H. 1998a. A review of Scolytodes Ferrari (Coleoptera: Scolytidae) associated with Cecropia (Cecropiaceae) in the northern Neotropics. Journal of Natural History, 32 (1), 31-84.

JORDAL, B.H. 1998b. New species of Scolytodes (Coleoptera: Scolytidae) from Costa Rica and Panama. Revista de Biología Tropical, 46, 407-419.

KIRKENDALL, L.R. 2017. Beetles (Coleoptera) of Peru: a survey of the families, Curculionidae: Platypodinae. The Coleopterists Bulletin, 71 (1), 99-115.

KIRKENDALL, L.R., y JORDAL, B.H. 2006. The bark and ambrosia beetles (Curculionidae, Scolytinae) of Cocos Island, Costa Rica and the role of mating systems in island zoogeography. Biological Journal of the Linnean Society, 89, 729-743.

KIRKENDALL, L.R.., y ØDEGAARD, F. 2007. Ongoing invasions of old-growth tropical forests: establishment of three incestuous beetle species in Central America (Curculionidae, Scolytinae). Zootaxa, 1588, 53-62.

KNIGHT, D.H. 1975. A Phytosociological Analysis of Species-Rich Tropical Forest on Barro Colorado Island, Panama. Ecological monographs, 45(3), 259-284.

LEIGH, E.G. 1999. Tropical Forest Ecology: a view from Barro Colorado Island. New York: Oxford University Press. 245 pp.

LEIGH, E.G. y WRIGHT, S.J. 1990. Barro Colorado Island and tropical biology. En: Four Neotropical rainforests. Ed: A.H. Gentry. p. 28-47. Connecticut: Yale University Press.

PECK, S.B. 2009. The beetles of Barbados, West Indies (Insecta: Coleoptera): diversity, distribution and faunal structure. Insecta Mundi, 0073, 1-51.

PECK, S.B. y THOMAS, M.C. 1998. Arthropods of Florida and neighboring land areas: a distributional checklist of the beetles (Coleoptera) of Florida, volumen 16. $180 \mathrm{pp}$.

PÉREZ DE LA CRUZ, M., EQUIHUA MARTÍNEZ, A., ROMERO NÁPOLES, J., SÁNCHEZ SOTO, S., GARCÍA LÓPEZ, E. y BRAVO MOJICA, H. 2009 b. Escolítidos (Coleoptera: Scolytidae) asociados al agroecosistema Cacao en Tabasco, México. Neotropical Entomology, 38 (5), 602-609.

PETROV, A.V. y MANDELSHTAM, M.Y. 2009. New data on ambrosia-beetles of the genus Sampsonius Eggers, 1935 with descriptions of two new species from Peru (Coleoptera: Curculionidae: Scolytinae). Koleopterologische Rundschau, 79, 313-319. 
PISTONE, D., GOHLI, J. y JORDAL, B.H. (2017). Molecular phylogeny of bark and ambrosia beetles (Curculionidae: Scolytinae) based on 18 molecular markers. Systematic Entomology, DOI: 10.1111/syen.12281, 1-20

PLOETZ, R.C., HULCR, J., WINGFIELD, M.J., y DE BEER, Z.W. 2013. Destructive tree diseases associated with ambrosia and bark beetles: black swan events in tree pathology?. Plant Disease, 97(7), 856-872.

REICHARDT, H. 1965. Scolytoidea (Coleoptera) 5: notes on Neotropical Platypodidae, mainly from Central America. Psyche, 72 (2), 159-166.

RODRIGUEZ O.R. y BARRIOS, H. 2017. Diversidad, distribución vertical y temporal de los escarabajos ambrosiales Platypodinae (Coleoptera: Curculionidae) en la Isla Barro Colorado, Panamá. Scientia, 27(2), 69-85.

RODRIGUEZ-FLORES, O. 2019. New records of Scolytinae from Nicaragua (Coleoptera, Curculionidae) with description of male genitalia of Taurodemus sharpi (Blandford, 1898). Revista Nicaragüense de Entomología, 166, 1-12.

SALGADO L, C.A. 2008. Análisis de abundancia y diversidad de Scolytinae (Coleoptera Curculionidae) en la zona del Canal de Panamá. Tesis Magister, Universidad de Panamá, Panamá. 62 pp.

SCHEDL, K.E. 1972. Monographie der Famile Platypodidae (Coleoptera). Junk, The Hague. 322 pp.

SMITH, S.M. y COGNATO, A.I. 2010. A taxonomic revision of Camptocerus Dejean (Coleoptera: Curculionidae: Scolytinae). Insecta Mundi, 0148: 1-88.

SOTO, H.M., GARCÍA, O., y CARBAJAL, C. 2013. Fauna de Curculionidae (Coleoptera) en huertas de aguacate Hass (Persea americana Mill) en Xalisco, Nayarit. Dugesiana, 20(2), 93-98.

STAINES, C.L. 1982. Distributional records of Platypodidae (Coleoptera) in Maryland. Proceedings of the Entomological Society of Washington, 84, 858-859.

ULYSHEN, M.D. y WAGNER, T.L. 2013. Quantifying arthropod contributions to wood decay. Methods in Ecology and Evolution, 4 (4), 345-352.

WINDSOR, D.M. 1990. Climate and Moisture Variability in a Tropical Forest: Longterm Records from Barro Colorado Island, Panamá. Smithsonian Contribution to the Earth Sciences. Washington: Smithsonian Institution Press. $145 \mathrm{pp}$.

WOOD, S.L. 1961. New records and species of Scolytidae (Coleoptera) from Colombia. Great Basin Naturalist, 21 (1), 1-7.

WOOD, S.L. 1966. New records and species of neotropical Platypodidae (Coleoptera). Great Basin Naturalist, 26 (3-4), 45-70. 
WOOD, S.L. 1967. New records and species of Neotropical bark beetles (Scolytidae: Coleoptera), Part II. Great Basin Naturalist, 27 (3), 119-141.

WOOD, S.L. 1971. New records and species of neotropical bark beetles (Scolytidae: Coleoptera), Part V. Brigham Young University Science Bulletin, Biological Series, 15 (3), 1-54.

WOOD, S.L. 1974. New Species of American Bark Beetles (Coleoptera: Scolytidae). Brigham Young University Science Bulletin, 19 (1), 1-73.

WOOD, S.L. 1975. New synonymy and new species of American bark beetles (Coleoptera: Scolytidae). Great Basin Naturalist, 35 (1), 21-32.

WOOD, S.L. 1978. New Synonymy and new species of American Bark Beetles (Coleoptera: Scolytidae), Part VII. Great Basin Naturalist, 38 (4), 397-405.

WOOD, S.L. 1982. The bark and ambrosia beetles of North and Central America (Coleoptera: Scolytidae), a taxonomic monograph. Great Basin Naturalist Memoirs, (6), 1-1356.

WOOD, S.L. 1985. New synonymy and new species of bark beetles (Coleoptera: Scolytidae). Great Basin Nat., 45 (2), 266-275.

WOOD, S.L. 1986. A reclassification of the genera of Scolytidae (Coleoptera). Great Basin Naturalist Memoirs, 10, 1-126.

WOOD, S.L. 1993. Revision of the genera of Platypodidae (Coleoptera). Great Basin Naturalist, 53, 259-281.

WOOD, S.L. 2007. Bark and Ambrosia beetles of South America (Coleoptera, Scolytidae). Monte L. Bean Life Science Museum, Brigham Young University. $900 \mathrm{pp}$.

WOOD, S.L. y BRIGHT, D.E. 1992. A catalog of Scolytidae and Platypodidae (Coleoptera), Part 2: Taxonomic Index. Great Basin Naturalist Memoirs, 13, 11553.

WOOD, S.L., STEVENS, G.C. y LEZAMA, H.J. 1991a. Los Scolytidae de Costa Rica: clave de géneros y de la subfamilia Hylesinae (Coleoptera). Revista de Biología Tropical, 39, 125-148.

WOOD, S.L., STEVENS, G.C. y LEZAMA, H.J. 1991b. Scolytidae (Coleoptera) de Costa Rica II: clave para la subfamilia Scolytinae, tribus: Scolytini, Ctenophorini, Micracini, Ipini, Dryocoetini, Xyleborini y Cryphalini. Revista de Biología Tropical, 39, 279-306.

WOOD, S.L., STEVENS, G.C. y LEZAMA, H.J. 1992. Los Scolytidae (Coleoptera) de Costa Rica. Clave de la subfamilia Scolytinae, Tribu: Corthylini. Revista de Biología Tropical, 40, 247-286. 


\section{ZANUNCIO, A.J.V., PASTORI, P.L., KIRKENDALL, L.R., LINO-NETO, J., SERRÃO,}

J.E. y ZANUNCIO, J.C. 2010. Megaplatypus mutatus (Chapuis) (Coleoptera: Curculionidae: Platypodinae) attacks hybrid Eucalyptus L'Héritier De Brutelle clones in Southern Espírito Santo, Brazil. The Coleopterists Bulletin, 64(1), 8183.

\section{AGRADECIMIENTOS}

A las siguientes instituciones, que cada una de ellas realizaron aportes imprescindibles para realizar la presente investigación: al Servicio Alemán de Intercambio Académico (DAAD) por el apoyo financiero; al Programa Centroamericano de Maestría en Entomología de la Universidad de Panamá por el préstamo de instalaciones, equipos y colecciones entomológicas y al Instituto Smithsoniano de Investigaciones Tropicales de Panamá por permitir el acceso al material entomológico de Scolytinae y Platypodinae. 